\title{
Estimation of Distributed Parameters in Permittivity Models of Composite Dielectric Materials Using Reflectance
}

\author{
H.T. Banks, Jared Catenacci and Shuhua Hu \\ Center for Research in Scientific Computation \\ North Carolina State University \\ Raleigh, NC 27695-8212 USA
}

August 31, 2014

\begin{abstract}
We investigate the feasibility of quantifying properties of a composite dielectric material through the reflectance, where the permittivity is described by the Lorentz model in which an unknown probability measure is placed on the model parameters. We summarize the computational and theoretical framework (the Prohorov metric framework) developed by our group in the past two decades for nonparametric estimation of probability measures using a least-squares method, and point out the limitation of the existing computational algorithms for this particular application. We then improve the algorithms, and demonstrate the feasibility of our proposed methods by numerical results obtained for both simulated data and experimental data for inorganic glass when considering the resonance wavenumber as a distributed parameter. Finally, in the case where the distributed parameter is taken as the relaxation time, we show using simulated data how the addition of derivative measurements improves the accuracy of the method.
\end{abstract}

Key Words: Electromagnetic interrogation or inverse problems, nonparametric leastsquares estimation, composite materials, reflection coefficient, wavenumbers, Prohorov metric framework, inorganic glass. 


\section{Introduction}

Complex materials such as ceramic matrix composites (CMC's) are currently being studied for their use in a wide range of applications, e.g., use in components of high temperature engines [1,34]. Simultaneously, noninvasive tools are being developed for use in characterizing damage and degradation of these materials. One such process which is popular is Fourier Transform Infrared (FTIR) spectroscopy, which has been shown to have the potential as a tool to investigate the oxidation behavior and general degradation of CMC's $[21,31,33,35]$. Additionally, reflectance spectroscopy has been shown to have sensitivity to heat treated ceramic thermal barrier coatings, which are being investigated for their use in turbine engines $[20,26]$. In this work, we explore the ability to use measured spectral responses obtained from a FTIR spectrometer for a general nonmagnetic dielectric material in order to quantify parameters in the composite permittivity model. Accurate quantification of these estimated parameters may prove to be a useful tool in the development of systems designed to monitor material damage and/or degradation.

Typically one would assume a particular combination of polarization models (Debye, Lorentz, Gaussian, etc.) with a predetermined number of dielectric parameters [4-6, 11]. However, in practice, generally the number of dielectric mechanisms are unknown, particularly for complex, highly heterogenous materials. In a case where the material under study is inorganic glass, to alleviate this difficulty, a convolution of the Lorentz and Gaussian functions (a linear combination of normal distributions is imposed on the resonance frequency in the Lorentz model) was proposed by Efimov in 1979 (e.g., see [24,25]). Our approach in the current effort is to allow for particular parameters, specifically, either the resonance frequencies or the relaxation times, to take a distributional form by imposing unknown probability measures on these parameters. Our goal is to place the weakest possible assumptions on the form of the underlying probability measure. Thus, we are interested in nonparametrically estimating the probability measures. Following popular conventions we will not always distinguish between probability measures and their associated cumulative distribution functions (or simply referred to as distributions) in the discussions below.

In the remainder of this presentation, we first give an introduction to the model describing the composite relative permittivity of a nonmagnetic dielectric medium which can be described by a generalized Lorentz polarization model, as well as the reflectance that is readily observed in practice. We then summarize the computational framework, the Prohorov Metric Framework (PMF), developed by our group in the past two decades to nonparametrically estimate a probability measure in the absence of individual longitudinal population data [12, Chap. 5], and point out the limitations of the existing computational algorithm for our problem. We then describe improvements to this algorithm, and demonstrate the capability of our proposed algorithm to accurately recover a probability measure which was used to generate the simulated data set. We also successfully apply this algorithm to estimate an unknown probability measure with experimental data. 


\section{The Model for the Complex Dielectric Constant and the Reflection Coefficient}

The Lorentz model is derived by considering the polarization which results from the displacement of electrons from equilibrium under the effect of an applied electromagnetic field. The Lorentz model for the relative permittivity with a single-resonance is given by

$$
\widehat{\varepsilon}_{r}(\omega)=\varepsilon_{\infty}-\frac{\omega_{p}^{2}}{\omega^{2}-i \omega / \tau_{f}-\omega_{0}^{2}} .
$$

In the above equation, $\varepsilon_{\infty}$ denotes the relative permittivity of the medium at infinite frequency, $\tau_{f}$ is the relaxation time, $i=\sqrt{-1}$ is the imaginary unit, and $\omega_{p}=\omega_{0} \sqrt{\varepsilon_{s}-\varepsilon_{\infty}}$ is called the plasma frequency of the medium, where $\omega_{0}$ is the resonance frequency, and $\varepsilon_{s}$ is the relative permittivity of the medium at zero frequency, also known as the "static" dielectric constant.

In practice it is typical for the data to be collected as a function of $k$, the wavenumber, rather than frequency. Using the relationship that $k=\omega /(2 \pi c)$, where $c$ is the speed of light in $\mathrm{cm} / \mathrm{s}$, we obtain the relative permittivity as a function of wavenumber

$$
\widehat{\varepsilon}_{r}(k)=\varepsilon_{\infty}-\frac{k_{p}^{2}}{k^{2}-i k / \tau_{k}-k_{0}^{2}} .
$$

In the above equation $k_{p}=k_{0} \sqrt{\varepsilon_{s}-\varepsilon_{\infty}}, k_{0}=\omega_{0} /(2 \pi c)$, and $\tau_{k}=2 \pi c \tau_{f}$. We will refer to $k_{0}$ as the resonance wavenumber and we will omit the subscript on the relaxation time $\tau_{k}$ when it is clear that we are referring to the relaxation time for the permittivity in terms of wavenumber.

In a homogeneous medium one might assume that the resonance wavenumbers $k_{0}$ or the relaxation times $\tau$ would be the same throughout the material particles, but for a composite material these would vary across the population of material particles, thus requiring some type of distribution on these resonance wavenumbers or relaxation times in order to characterize the heterogeneous population of particles. To allow for a distribution $G$ of either resonance wavenumbers, or relaxation times, over an admissible set $\mathcal{K} \subset \mathbb{R}$, we generalize the relative permittivity for the Lorentz model (2.2) to be

$$
\widehat{\varepsilon}_{r}(k ; G, \theta)=\varepsilon_{\infty}-\int_{\mathcal{K}} \frac{k_{p}^{2}}{k^{2}-i k / \tau-k_{0}^{2}} d G,
$$

where $G \in \mathbb{P}(\mathcal{K})$, the set of admissible probability measures on $\mathcal{K}$. In the case of assuming a distribution of resonance wavenumbers we take $\theta=\left(\varepsilon_{s}, \varepsilon_{\infty}, \tau\right)^{T} \in \Theta \subset \mathbb{R}^{3}$ and in the case where there is a distribution on the relaxation times we take $\theta=\left(\varepsilon_{s}, \varepsilon_{\infty}, k_{0}\right)^{T} \in \Theta \subset \mathbb{R}^{3}$ with $\Theta$ assumed to be compact for either case. Thus for our composite materials the relative

permittivity $\widehat{\varepsilon}_{r}(k)=\varepsilon_{\infty}-\frac{k_{p}^{2}}{k^{2}-i k / \tau_{k}-k_{0}^{2}}$ of $(2.2)$ is replaced by $\widehat{\varepsilon}_{r}(k ; G, \theta)=\varepsilon_{\infty}-\int_{\mathcal{K}} \frac{k_{p}^{2}}{k^{2}-i k / \tau-k_{0}^{2}} d G$ of (2.3).

We next turn our attention to obtaining a model for the reflectance. For simplicity, we assume that a monochromatic uniform wave of wavenumber $k$ is normally incident on a plane interface between free space and a dielectric medium. In addition, we assume that the 
electric field is polarized perpendicular to the plane of incidence. With these assumptions the fully complex reflection coefficient is given by

$$
r(k ; G, \theta)=\frac{1-\sqrt{\widehat{\varepsilon}_{r}(k ; G, \theta)}}{1+\sqrt{\widehat{\varepsilon}_{r}(k ; G, \theta)}} .
$$

The derivation of the reflection coefficient can be found in many electromagnetic treatments (e.g., see [6], [17, Section 9.3], [29], [36]). An interferometer does not measure the reflection coefficient directly, rather the observable is the reflectance

$$
R(k ; G, \theta)=|r(k ; G, \theta)|^{2} .
$$

We remark that some interferometers have the capability to obtain the derivative of the reflectance. Specifically, the Bruker $80 \mathrm{~V}$ two beam interferometer [18] is capable of calculating derivatives up to the fifth order using the Savitzky-Golay algorithm. This algorithm smoothes the data through fitting successive sub-sets of adjacent data points with a low-degree polynomial. Hence, we will also explore the use of derivative of the reflectance measurements, where the derivative is taken with respect to the wavenumber $k$. To simulate how the Bruker $80 \mathrm{~V}$ interferometer obtains derivative information, our derivative measurements will be obtained through the application of the Savitzky-Golay algorithm to the raw (simulated/experimental) reflectance data.

\section{Computational Framework}

In this section we examine an inverse problem methodology for estimating the probability measure $G$ as well as the additional model parameters $\theta$.

\subsection{Statistical Model}

We consider a statistical model of the form

$$
Y_{j}=h\left(k_{j} ; G_{0}, \theta_{0}\right)+\mathcal{V}_{j}, \quad j=0,1,2, \ldots, n
$$

In the above equation, $h\left(k_{j} ; G_{0}, \theta_{0}\right)$ corresponds to the observed part of the system with the "true" probability measure $G_{0}$ and "true" parameters $\theta_{0}$ at the measurement (input) wavenumber $k_{j}, \mathcal{V}_{j}$ denotes the measurement error at the measurement wavenumber $k_{j}$, and $n+1$ is the total number of observations. For simplicity in the current proof of concept discussion, we assume that $\mathcal{V}_{j}, j=0,1,2, \ldots, n$, are independent and identically distributed with zero mean and constant covariance matric $\sigma_{0}^{2} * I_{c}$, where $I_{c}$ is an $\ell \times \ell$ identity matrix with $\ell$ being the dimension of $h$.

We note here that the usual statistical concept of longitudinal (in time) data is in our problem replaced by input wavenumber data as opposed to the more commonly encountered notion of time point observations found in mathematical and statistical formulations. Our observations depend on the population level probability measures where we have only "aggregate" population particle data for the heterogeneous material as opposed to individual data. More specifically, we do not have $h\left(k_{j} ; \theta\right), j=1,2, \ldots, n$, which would constitute individual 
population (particle) data, i.e., we don't have data based on the particle permittivities $\widehat{\varepsilon}_{r}$ of (2.2) for individual material particles as a function of input wavenumbers $k_{j}$.

As discussed above, we consider two types of data. In the case that the observed part of the system is the reflectance, we have

$$
h\left(k_{j} ; G, \theta\right)=R\left(k_{j} ; G, \theta\right) .
$$

In the case in which the data contains both the reflectance and the derivative of the reflectance, $h$ has two components and is given by

$$
h\left(k_{j} ; G, \theta\right)=\left(R\left(k_{j} ; G, \theta\right), \frac{\frac{\partial}{\partial k} R\left(k_{j} ; G, \theta\right)}{\max _{j} \frac{\partial}{\partial k} R\left(k_{j} ; G, \theta\right)}\right)^{T} .
$$

We remark that the reason for choosing the second component of $h$ as above is because the magnitude of the derivative of the reflectance is extremely small compared to the value of the reflectance. Note that a closed form analytical solution for the derivative of the reflectance has proved to be very difficult to obtain due to the form of $R(k ; G, \theta)$. Hence, we use a simple forward difference scheme to approximate it.

\subsection{Inverse Problem}

Under the assumptions for the measurement errors in the statistical model, the estimator of $\left(G_{0}, \theta_{0}\right)$ can be obtained using the ordinary least squares formulation (e.g., see $[12,15]$ for details)

$$
\left(G^{n}, \theta^{n}\right)=\underset{(G, \theta) \in(\mathbb{P}(\mathcal{K}) \times \Theta)}{\arg \min _{j=0}} \sum^{n}\left(h\left(k_{j} ; G, \theta\right)-Y_{j}\right)^{T}\left(h\left(k_{j} ; G, \theta\right)-Y_{j}\right) .
$$

The corresponding realization of $\left(G^{n}, \theta^{n}\right)$ can be calculated through

$$
\left(\widehat{G}^{n}, \widehat{\theta}^{n}\right)=\underset{(G, \theta) \in(\mathbb{P}(\mathcal{K}) \times \Theta)}{\arg \min _{j=0}} \sum^{n}\left(h\left(k_{j} ; G, \theta\right)-y_{j}\right)^{T}\left(h\left(k_{j} ; G, \theta\right)-y_{j}\right),
$$

where $y_{j}$ is a realization of $Y_{j}$, that is,

$$
y_{j}=h\left(k_{j} ; G_{0}, \theta_{0}\right)+\nu_{j}, \quad j=0,1,2, \ldots, n,
$$

with $\nu_{j}$ being a realization of $\mathcal{V}_{j}$.

The existence of a minimizer to (3.4) or (3.5) can be established under the Prohorov metric framework as developed in [2,3,10-14] specifically to use with population level (aggregate) data as opposed to individual longitudinal data. The Prohorov metric was introduced in [32] and is defined as follows.

Definition 3.1. Let $\mathbb{F} \subset \mathcal{K}$ be any closed set and define $\mathbb{F}^{\epsilon}=\left\{\xi \in \mathcal{K}: \inf _{\tilde{\xi} \in \mathbb{F}} d(\xi, \tilde{\xi})<\epsilon\right\}$, where $d$ denotes the metric on $\mathcal{K}$. For $G, Q \in \mathbb{P}(\mathcal{K})$, the Prohorov metric is given by

$$
\rho(G, Q)=\inf \left\{\epsilon>0 \mid Q(\mathbb{F}) \leq G\left(\mathbb{F}^{\epsilon}\right)+\epsilon \text { and } G(\mathbb{F}) \leq Q\left(\mathbb{F}^{\epsilon}\right)+\epsilon, \text { for all } \mathbb{F} \text { closed in } \mathcal{K}\right\}
$$


It is clear from the definition above that the meaning of the Prohorov metric is far from intuitive. Yet one can provide several useful characterizations. For example, convergence in the Prohorov metric is equivalent to the weak* convergence if we view $\mathbb{P}(\mathcal{K}) \subset C_{B}^{*}(\mathcal{K})$, where $C_{B}^{*}(\mathcal{K})$ denotes the topological dual of the space $C_{B}(\mathcal{K})$ of bounded and continuous functions on $\mathcal{K}$. In other words, the statement $\rho\left(G_{j}, G\right) \rightarrow 0$ is equivalent to the statement

$$
\int_{\mathcal{K}} h(\xi) d G_{j}(\xi) \rightarrow \int_{\mathcal{K}} h(\xi) d G(\xi) \text { for any } h \in C_{B}(\mathcal{K}) .
$$

The Prohorov metric also possesses many useful and important properties. For example, if we assume that $\mathcal{K}$ is compact, then $\mathbb{P}(\mathcal{K})$ is a compact metric space when taken with the Prohorov metric $\rho$. We refer interested readers to $[2,12,16,22]$ for more information on the Prohorov metric and its properties.

Note that $h$ is continuous with respect to $G$ and $\theta$. Hence, based on the above discussion we know that if both $\mathcal{K}$ and $\Theta$ are assumed to be compact, then there exists a solution to (3.4) or (3.5). Under usual standard assumptions on $h$ and how data is sampled, the consistency of estimators can also be established (see our companion theoretical paper [7] for details).

We note that (3.5) is an infinite-dimensional optimization problem $($ as $\mathbb{P}(\mathcal{K})$ is an infinitedimensional space). Hence, we need to approximate the infinite-dimensional space $\mathbb{P}(\mathcal{K})$ with finite-dimensional spaces $\mathbb{P}^{N}(\mathcal{K})$ so that we have a computationally tractable finitedimensional optimization problem given by

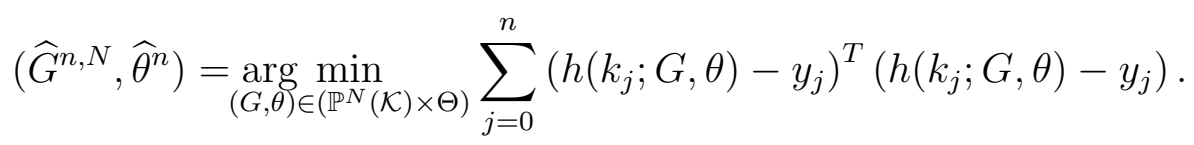

Of course, one needs to choose $\mathbb{P}^{N}(\mathcal{K})$ in a meaningful way so that $\widehat{G}^{n, N}$ approaches $\widehat{G}^{n}$ as $N$ goes to infinity. One such approximation method involves using Dirac measures to approximate the probability measure and can be used regardless of the smoothness of the underlying desired probability measures. This is especially useful in the situations where one has no knowledge of the sought-after probability measures. The theoretical foundation for such an approximation relies on the Prohorov metric framework and the following theorem (see [3] for details).

Theorem 3.2. Assume $\mathcal{K} \subset \mathbb{R}$ is compact. Let $\mathcal{K}_{D}=\left\{\xi_{j}\right\}_{j=1}^{\infty}$ be an enumeration of a countable dense subset of $\mathcal{K}$, and $\Delta_{\xi_{j}}$ be the Dirac measure with atom at $\xi_{j}$. Define

$$
\tilde{\mathbb{P}}_{D}(\mathcal{K})=\left\{G \in \mathbb{P}(\mathcal{K}) \mid G=\sum_{j=1}^{N} a_{j} \Delta_{\xi_{j}}, \xi_{j} \in \mathcal{K}, a_{j} \in[0,1] \cap \mathbb{Q}, \sum_{j=1}^{N} a_{j}=1, N \in \mathbb{N}\right\},
$$

where $\mathbb{Q} \subset \mathbb{R}$ denotes the set of all rational numbers. Then $\tilde{\mathbb{P}}_{D}(\mathcal{K})$ is dense in $\mathbb{P}(\mathcal{K})$.

With this approximation, the least squares problem that we wish to solve is (3.7) with

$$
\mathbb{P}^{N}(\mathcal{K})=\left\{G \in \mathbb{P}(\mathcal{K}) \mid G=\sum_{j=1}^{N} \alpha_{j} \Delta_{\xi_{j}}, \text { where } \alpha_{j} \geq 0 \text { and } \sum_{j=1}^{N} \alpha_{j}=1\right\} .
$$


Under this computational framework the optimization problem (3.7) is reduced to a standard optimization problem over $\mathbb{R}^{N+3}$ in which we seek to estimate the finite set of values $\left\{\alpha_{m}\right\}_{m=1}^{N} \cup\{\theta\}$.

We remark that the Dirac measure approximation method has been successfully used to estimate probability measures in a number of applications (e.g., see [9-11,14]). However, it was noted in practice that a poor choice of nodes and improper number of nodes could both result in ill-conditioned inverse problems. As we shall see in the next section, this is also discerned in the problem we investigate here. To alleviate some of these difficulties, we propose to estimate both weights and nodes. The feasibility of this modified method is demonstrated in the next section with both simulated data and experimental data.

\section{Numerical Results}

In this section we present results for obtaining an estimation of a probability measure on the resonance wavenumber using both simulated data and experimental data sets for inorganic glass (taken from [23]) where $h$ is given by (3.2). We begin by presenting results obtained using simulated data to explore the reliability and accuracy, as well as the limitations of the above approximation methods. We then improve these approximation methods by estimating both the weights and the nodes, and demonstrate the ability of our proposed methods to accurately recover a probability measure which was used to generate the simulated data set. Finally we use the proposed algorithm to estimate an unknown probability measure with experimental data sets.

In addition, we also give results in which the estimated probability measure is on the relaxation time rather than the resonance wavenumber. Using simulated data where $h$ is given by (3.2), we show in this case that estimating both the weights and the nodes is not sufficient to give reliable results. We then show how the the accuracy of the estimation of the probability measure can be improved by the use of derivative information. In order to obtain derivative data we use the Savitzky-Golay algorithm, which as was mentioned previously, is a common algorithm used by modern interferometers to obtain derivative measurements.

\subsection{Results Obtained Using Simulated Data When Estimating a Probability Measure on the Resonance Wavenumber}

In this section we will attempt to estimate a probability measure on the resonance wavenumber $k_{0}$ where $h$ is given by (3.2). For the remainder of this section we will take the vector of fixed parameters to be $\theta=\left(\varepsilon_{s}, \varepsilon_{\infty}, \tau\right)^{T}$ and we will denote the nodes of the Dirac masses as $\xi_{m}=k_{0_{m}}$. First, we will use the simulated data to discuss the importance of the placement of nodes of the Dirac measures, i.e., the values of $k_{0 j}, j=1,2, \ldots, N$. In all of the following results shown in this section, the data was generated by evaluating equation (3.6), where $k_{j}=400+10 j, j=0,1,2, \ldots 69$, the true probability measure $G_{0}=\sum_{m=1}^{3} \alpha_{m} \Delta_{k_{0 m}}$ with

$$
\alpha_{1}=\alpha_{2}=0.05, \quad \alpha_{3}=0.9, \quad k_{01}=570, \quad k_{02}=580, \quad k_{03}=850 .
$$

We used the true parameters $\theta_{0}=(2.7,1.9,0.03)^{T}$, and $\nu_{j}$ was chosen as a realization of a normally distributed random variable with mean 0 and standard deviation $\sigma_{0}=0.005$. 
To simplify the scenario, we first only estimate the weights of the Dirac measures, i.e., $\alpha_{m}, m=1,2, \ldots, N$, with $\theta_{0}$ assumed to be known. In Figure 1 we present the model fit and the estimated distribution where the nodes are chosen to be evenly spaced in the interval [405, 1080], where $N=25$. In this case the model and the estimated distribution are both an excellent fit to the data and the true distribution, respectively. However, by altering the node placement so that there are now 25 nodes evenly spaced in the interval $[405,1100]$, the fit to the data is not as good as in the previous case, and the estimated distribution also fails to be as good a fit as before. These results are shown in Figure 2. Clearly, from this simple example, we see that our ability to achieve good fits to the data and accurate estimations of the distribution relies on the placement of the nodes.
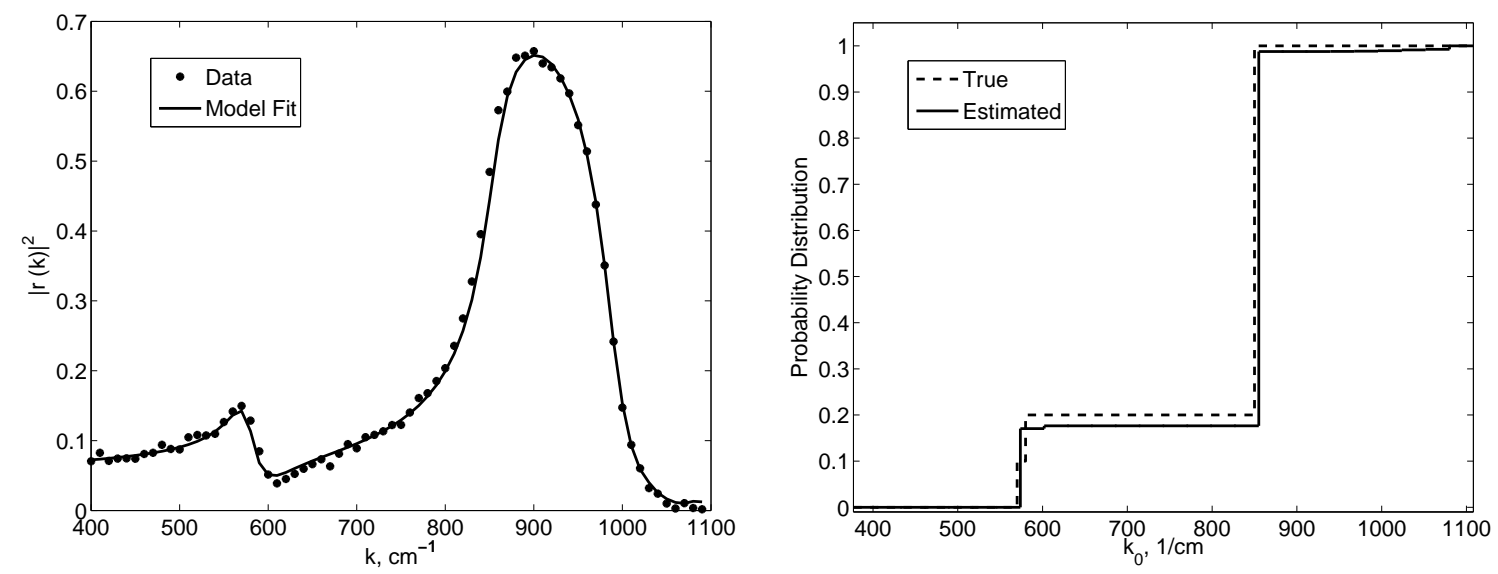

Figure 1: The model fit to the simulated data (left) and the estimated distribution of wavenumbers (right), where the nodes are evenly placed over $[405,1080]$ with $N=25$.
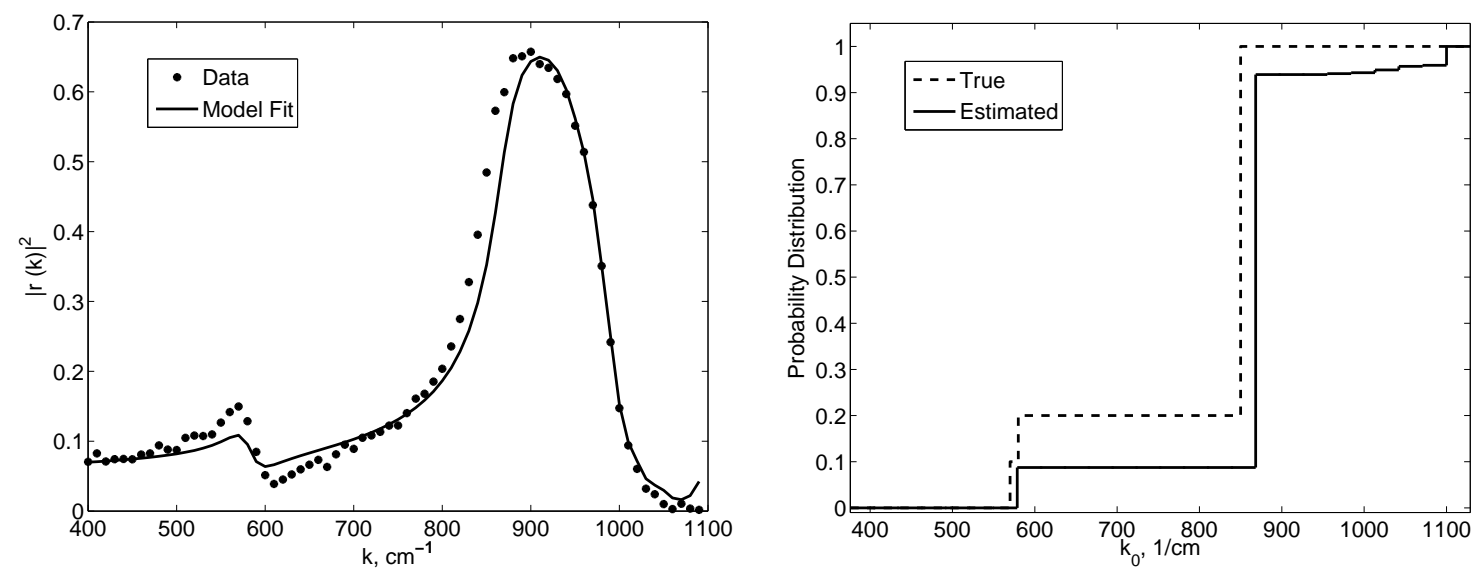

Figure 2: The model fit to the simulated data (left) and the estimated distribution of wavenumbers (right), where the nodes are evenly placed over [405, 1100] with $N=25$.

In attempts to alleviate these difficulties, we seek to also optimize the node locations as well as the weights of the Dirac measures. We did so by including the node locations as additional parameters in the minimization problem. Thus, we can reformulate the minimization 
problem as

$$
\left(\hat{\boldsymbol{\alpha}}^{N}, \hat{\mathbf{k}}_{0}^{N}\right)=\underset{\boldsymbol{\alpha} \in \mathbb{R}_{w}^{N}, \mathbf{k}_{0} \in \mathcal{K}^{N}}{\operatorname{argmin}} \sum_{j=0}^{n}\left(h\left(k_{j} ; G, \theta_{0}\right)-y_{j}\right)^{2},
$$

where $G=\sum_{j=1}^{N} \alpha_{j} \Delta_{k_{0 j}}$, and the weights and node locations are chosen respectively from

$$
\begin{aligned}
& \widetilde{\mathbb{R}}_{w}^{N}=\left\{\boldsymbol{\alpha}=\left(\alpha_{1}, \alpha_{2}, \ldots, \alpha_{N}\right)^{T} \mid \alpha_{j} \geq 0 \text { and } \sum_{j=1}^{N} \alpha_{j}=1\right\}, \\
& \mathcal{K}^{N}=\left\{\mathbf{k}_{0}=\left(k_{01}, k_{02}, \ldots, k_{0 N}\right)^{T} \mid k_{0 j} \in \mathcal{K}, j=1,2, \ldots, N\right\} .
\end{aligned}
$$

The estimated probability measure is then given by $\widehat{G}=\sum_{j=1}^{N} \hat{\alpha}_{j} \Delta_{\hat{k}_{0 j}}$, where $\hat{\alpha}_{j}$ and $\hat{k}_{0 j}$ are the $j$ th element of $\hat{\boldsymbol{\alpha}}^{N}$ and $\hat{\mathbf{k}}_{0}^{N}$, respectively. The results of this inverse problem are shown in Figure 3. In this case we see an excellent fit to the data, and the estimated distribution falls nearly precisely on the true distribution.
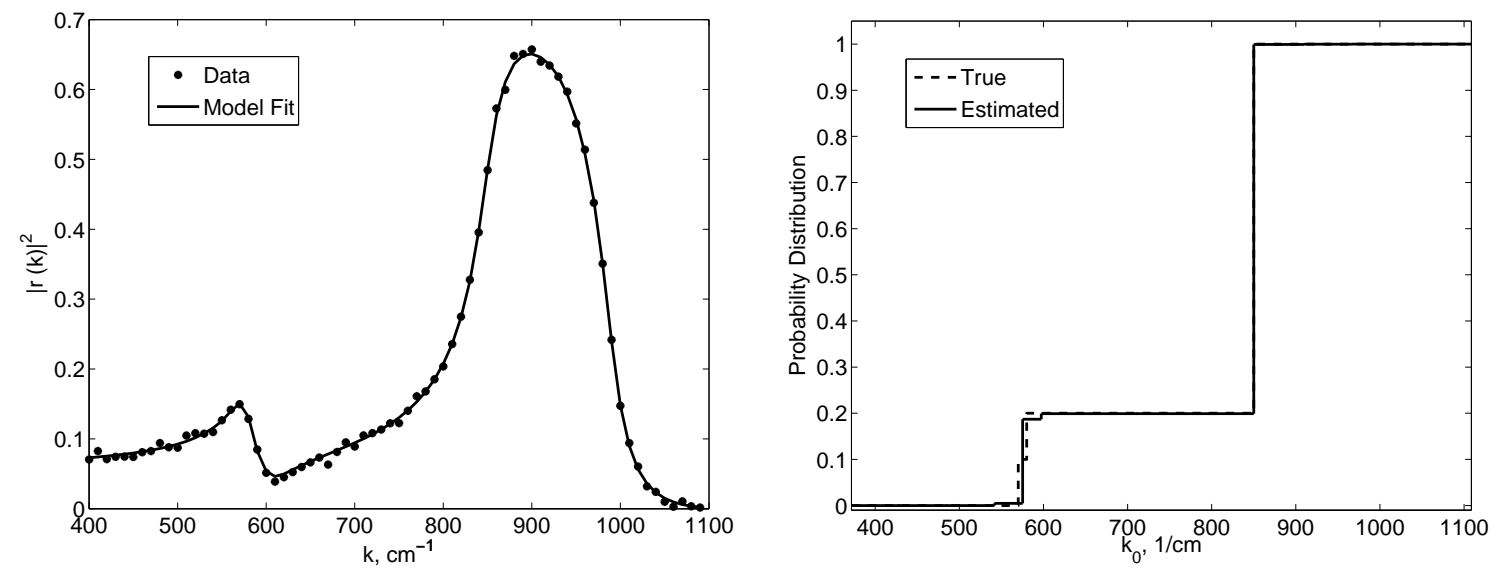

Figure 3: Model fit (left) and estimated distribution (right) where the parameters $\varepsilon_{s}, \varepsilon_{\infty}$ and $\tau$ are fixed and the weights and node locations were optimized with $N=25$.

Next we consider the case where the parameters $\varepsilon_{s}, \varepsilon_{\infty}$ and $\tau$ are not known, and thus must also be estimated; that is, our minimization problem is given by

$$
\left(\hat{\boldsymbol{\alpha}}^{N}, \hat{\mathbf{k}}_{0}^{N}, \widehat{\theta}\right)=\underset{\boldsymbol{\alpha} \in \mathbb{R}_{w}^{N}, \mathbf{k}_{0} \in \mathcal{K}^{N}, \theta \in \Theta}{\arg } \sum_{j=0}^{n}\left(h\left(k_{j} ; G, \theta\right)-y_{j}\right)^{2},
$$

where $G=\sum_{j=1}^{N} \alpha_{j} \Delta_{k_{0 j}}, \widetilde{\mathbb{R}}_{w}^{N}$ and $\mathcal{K}^{N}$ are defined by (4.2). In doing so, we impose the additional constraint that $\varepsilon_{s}>\varepsilon_{\infty}$. This was done to add stability to the inverse problem with respect to the initial guesses for the parameter values. The parameters and the distribution were estimated in this way for $N=5,10,15,20,25$, and 30, and the results are given in Table 1 and Figures 4-9. For all of the various number of nodes chosen, the model is an excellent fit to the data. For the cases when a low number of nodes is used, e.g., $N=5$ and 10 , the estimated distribution coincides with the true distribution, and the estimated values for parameters $\varepsilon_{s}, \varepsilon_{\infty}$ and $\tau$ are very close to their corresponding true values. As $N$ increases, we see that the estimated distributions begin to deviate from the true distribution and the 


\begin{tabular}{|c|ccc|}
\hline$N$ & $\varepsilon_{s}$ & $\varepsilon_{\infty}$ & $\tau(\mathrm{cm})$ \\
\hline 5 & 2.6749 & 1.8781 & 0.0298 \\
10 & 2.6703 & 1.8703 & 0.0297 \\
15 & 2.6618 & 1.8261 & 0.0303 \\
20 & 2.6563 & 1.8163 & 0.0306 \\
25 & 2.6713 & 1.8408 & 0.0341 \\
30 & 2.6661 & 1.8441 & 0.0389 \\
\hline True Values & 2.700 & 1.900 & 0.0300 \\
\hline
\end{tabular}

Table 1: Estimations obtained using the simulated data for various numbers of Dirac measures.

estimation accuracy for parameters $\varepsilon_{s}, \varepsilon_{\infty}$ and $\tau$ decreases. This is especially true for parameter $\tau$ : its relative error for the cases of $N=25$ and 30 is 0.14 and 0.30 respectively, and it is considerably higher than those obtained with a lower value of $N$ (for $N=5,10,15,20$ the mean relative error is 0.06). We remark that the loss of estimation accuracy as $N$ increases is in agreement with the common understanding that for a fixed number of observations the estimation accuracy in general decreases as the number of estimated parameters increases (e.g., see [7,19]). Actually, this is how model selection criteria play a role as all model selection criteria such as the Akaike Information Criterion and the Bayesian Information Criterion are based to some extent on the principle of parsimony (a balance between the model accuracy and the estimation accuracy). We again refer the interested reader to $[7,19]$ for more information on this. Using a model selection criteria to select a best value for $N$ is an area for current investigations [7].
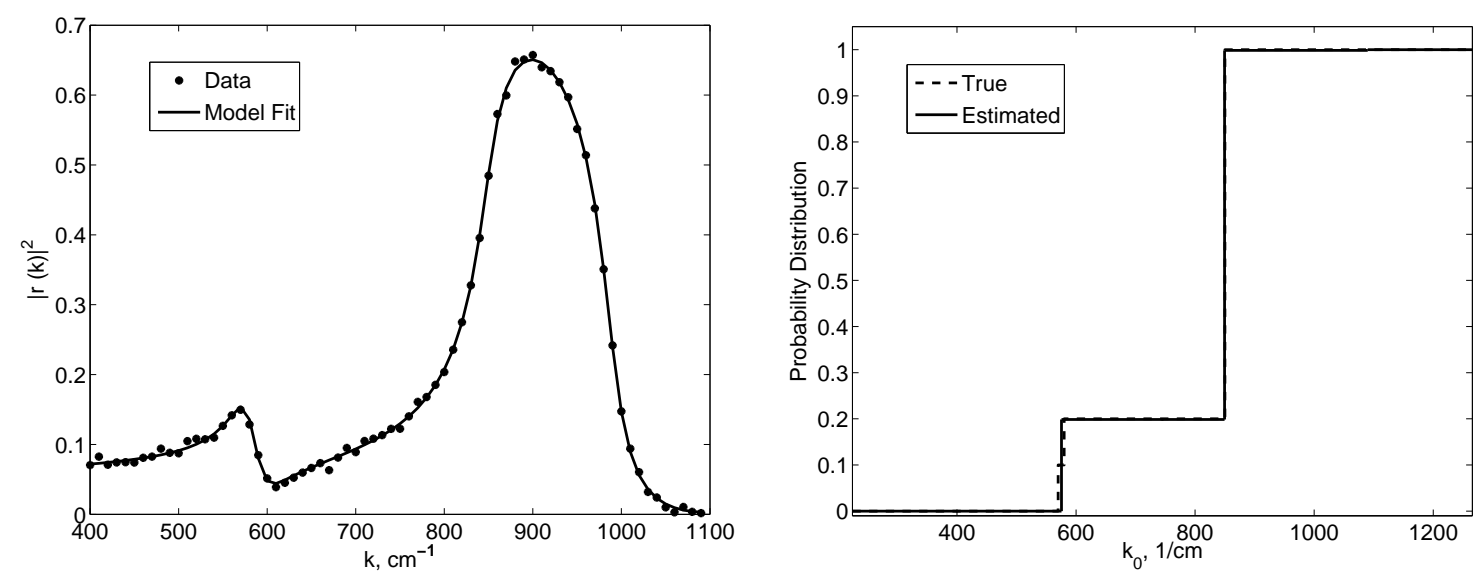

Figure 4: Model fit (left) and the estimated distribution (right) from the full inverse problem (4.3) where $N=5$. 

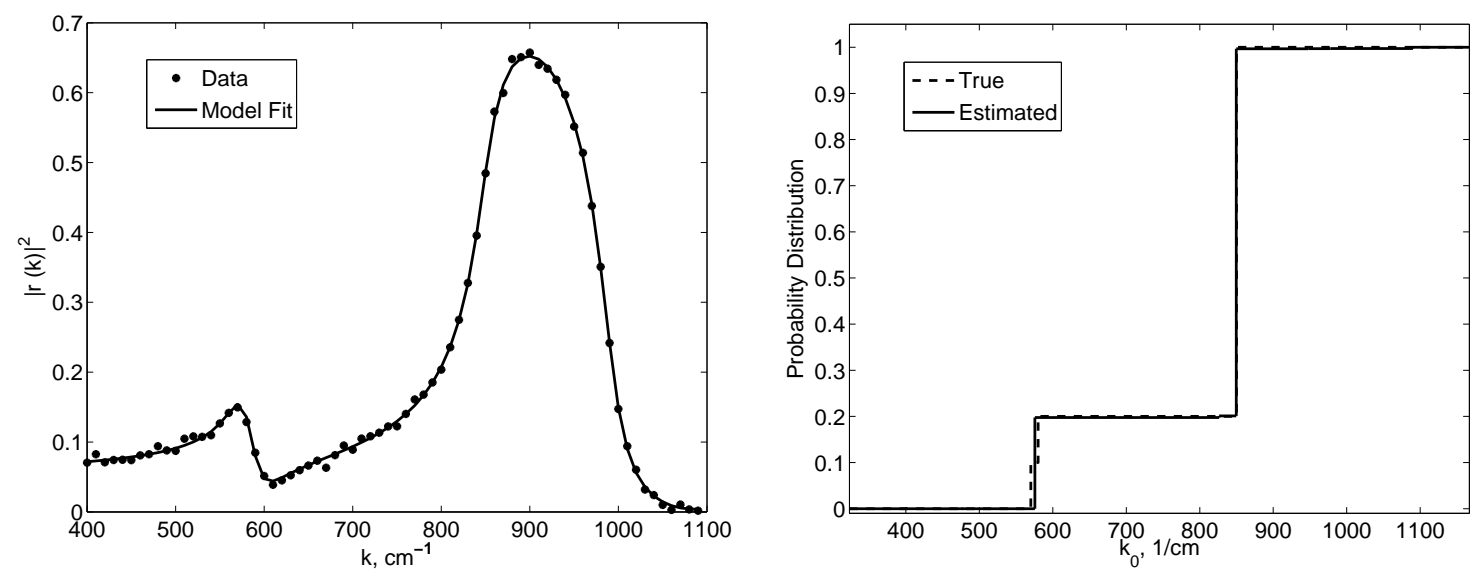

Figure 5: Model fit (left) and the estimated distribution (right) from the full inverse problem (4.3) where $N=10$.
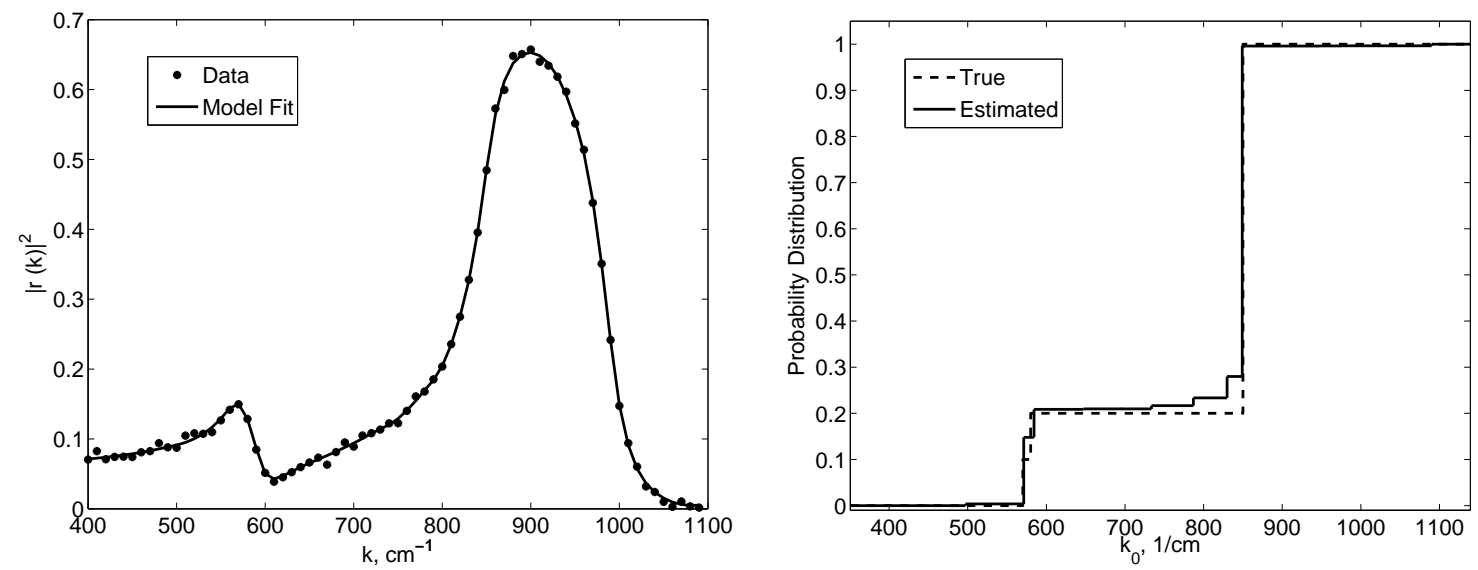

Figure 6: Model fit (left) and the estimated distribution (right) from the full inverse problem (4.3) where $N=15$.
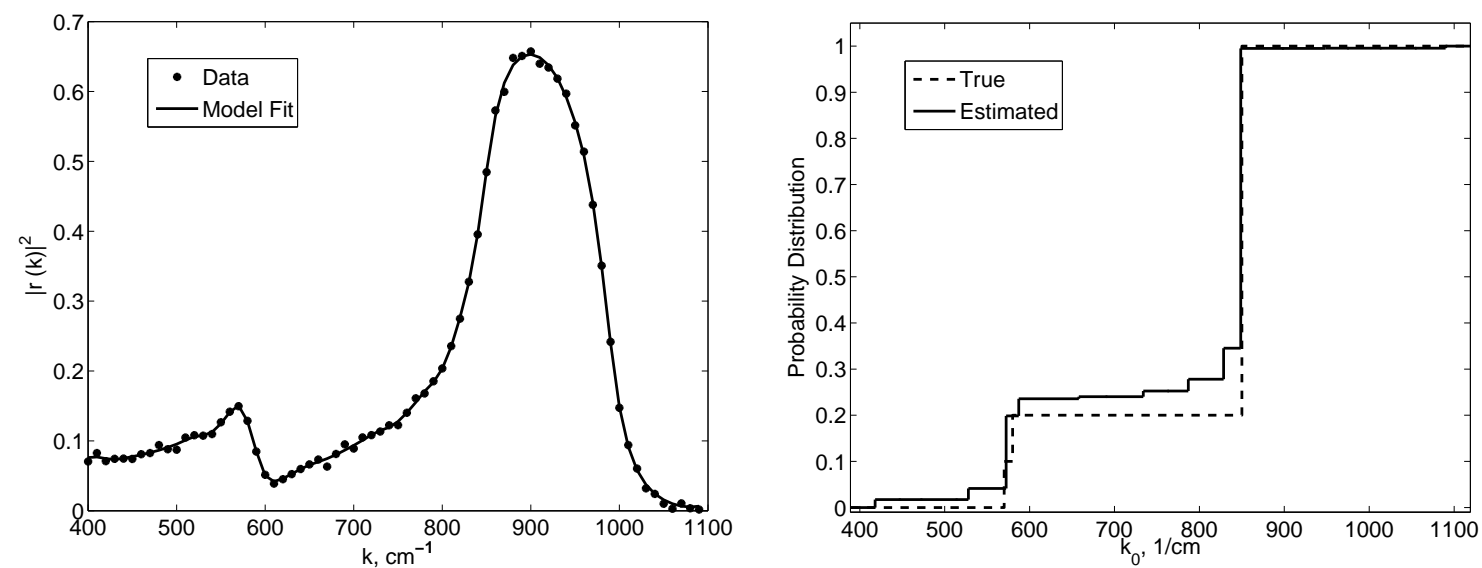

Figure 7: Model fit (left) and the estimated distribution (right) from the full inverse problem (4.3) where $N=20$. 

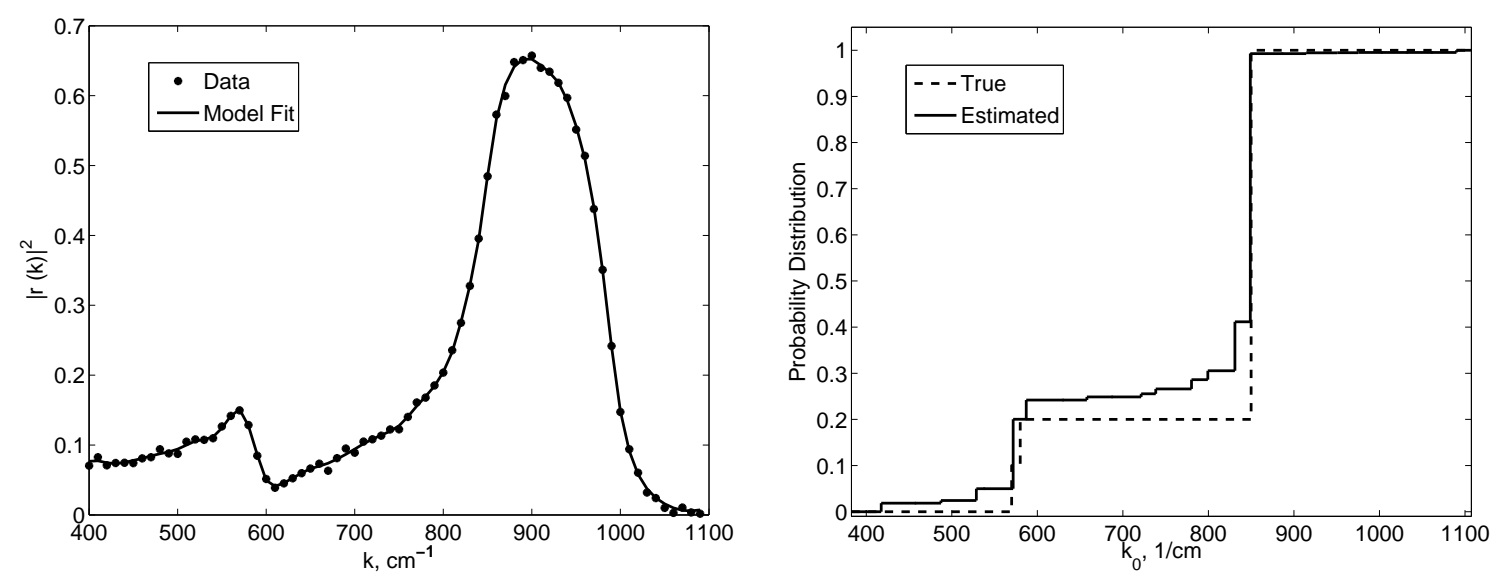

Figure 8: Model fit (left) and the estimated distribution (right) from the full inverse problem (4.3) where $N=25$.
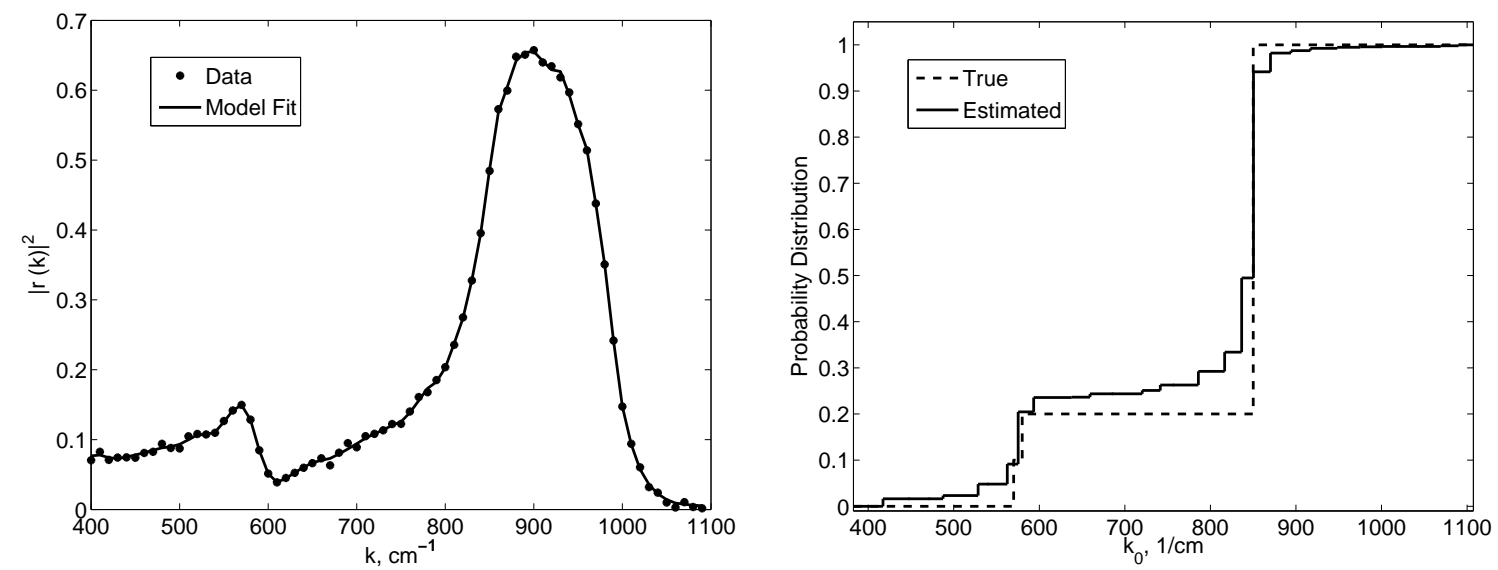

Figure 9: Model fit (left) and the estimated distribution (right) from the full inverse problem (4.3) where $N=30$.

\subsection{Results Obtained Using Inorganic Glass Data When Estimat- ing a Probability Measure on the Resonance Wavenumber}

To illustrate the feasibility of our approximation methods on experimental data sets, we present results obtained using inorganic glass data available in [23]. We first consider reflectance data collected from Vitreous Germania in the 400 to $1100 \mathrm{~cm}^{-1}$ range (see Table A7 in [23]). Using $N=5,10,15,20$ and 25 Dirac measures to estimate the distribution of resonance wavenumbers, the corresponding model fits and probability distribution estimations are given in Figures 10-14. Unlike when using the simulated data, we do see an improvement in the model fits as $N$ is increased. Recall that the simulated data was generated using a true distribution of only 3 discrete measures, 2 of which were very close to each other. Therefore it is not surprising that we were able to obtain very good model fits using only a small number of Dirac measures in that case. Here, it appears as if the unknown distribution for the Vitreous Germania data is made up of either many more discrete measures which are closely clustered in the 500-600, and the $800-900 \mathrm{~cm}^{-1}$ range, or a continuous distribution 
whose probability density function only has nontrivial values over those before mentioned ranges. If this is indeed the case, then it follows that more Dirac measures are needed in order to obtain an accurate fit to the data. Observe that in Figure 15 where we superimpose the estimated probability distributions obtained using the various values of $N$, we see that the distributions exhibit a similar basic structure (with increases from 0 to $\sim 0.2-0.3$ over the 500-600 range, and a second increase to $\sim 1$ over the 800-900 range).
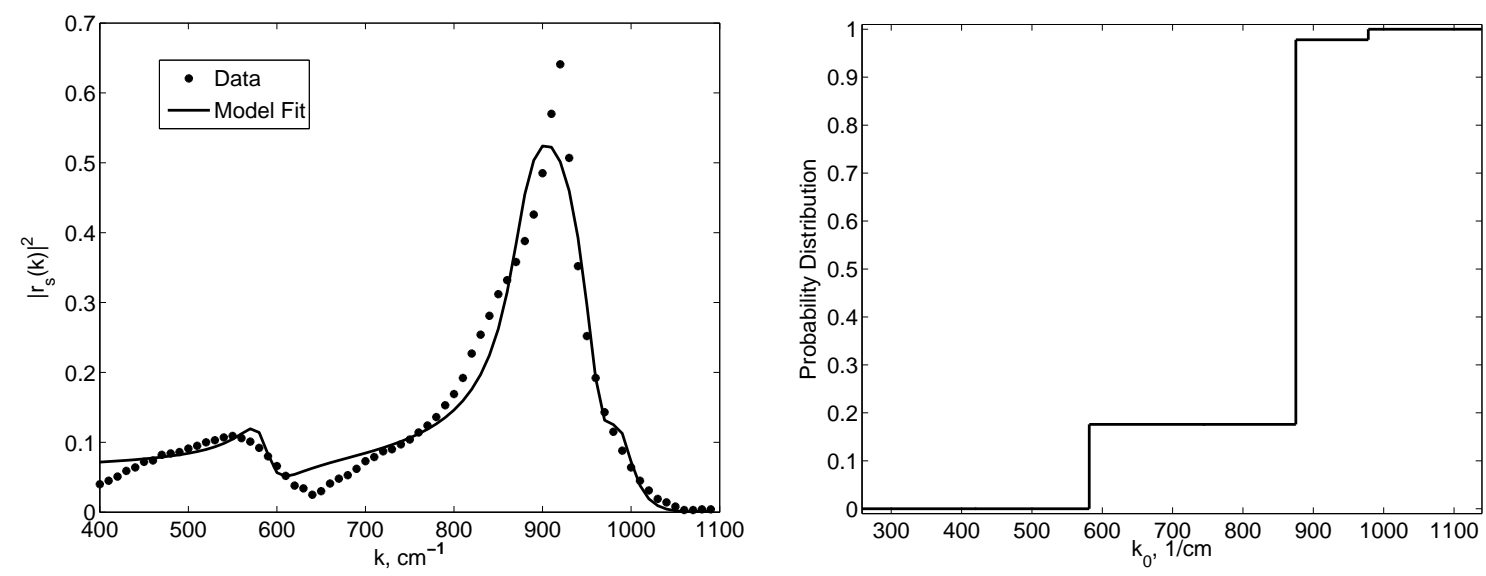

Figure 10: Model fit (left) and the estimated distribution (right) from the full inverse problem (4.3) where $N=5$ for Vitreous Germania.
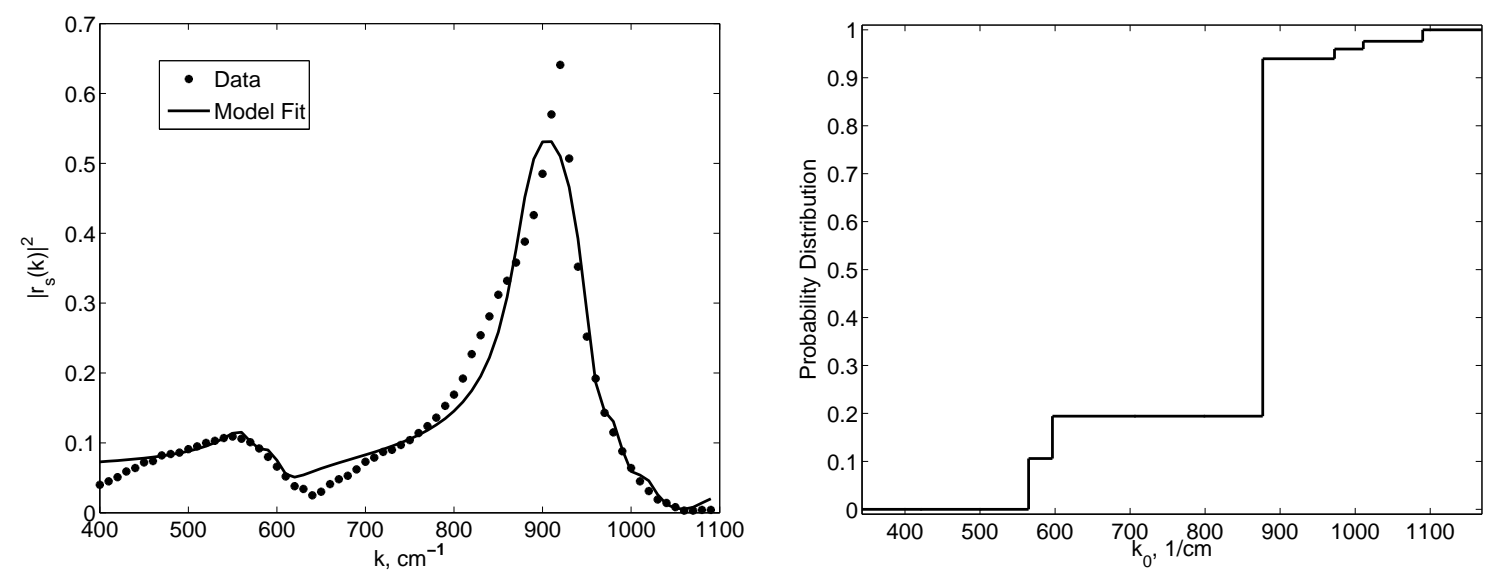

Figure 11: Model fit (left) and the estimated distribution (right) from the full inverse problem (4.3) where $N=10$ for Vitreous Germania. 

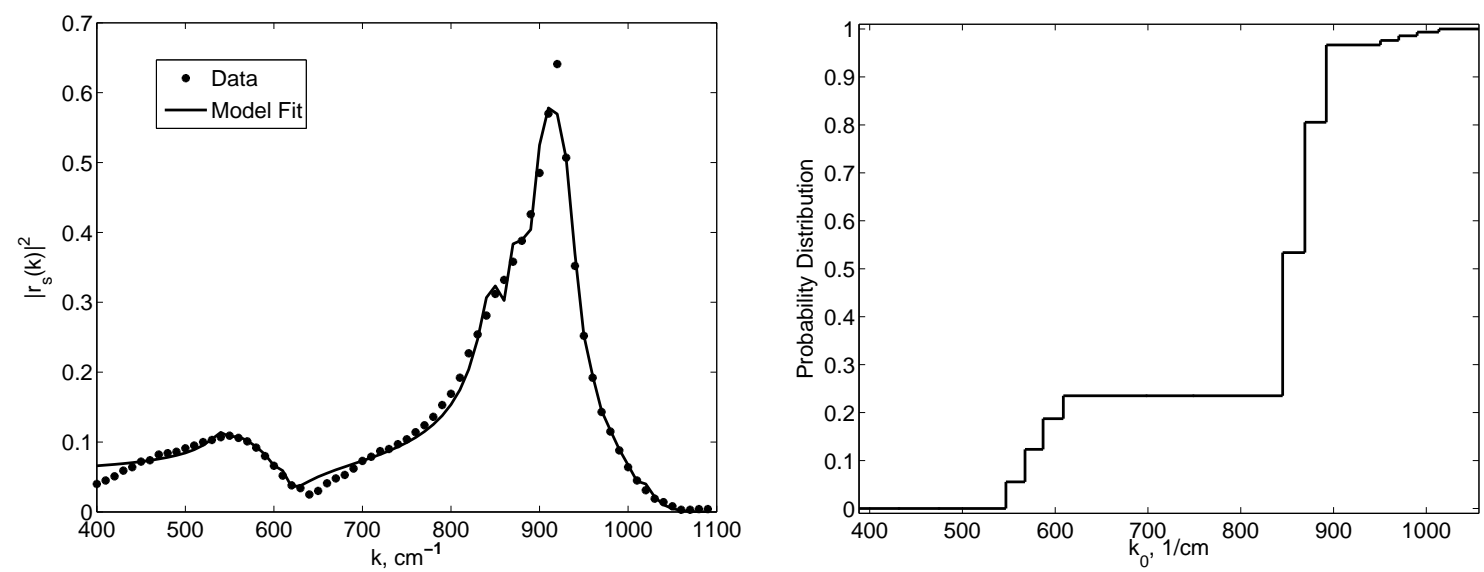

Figure 12: Model fit (left) and the estimated distribution (right) from the full inverse problem (4.3) where $N=15$ for Vitreous Germania.
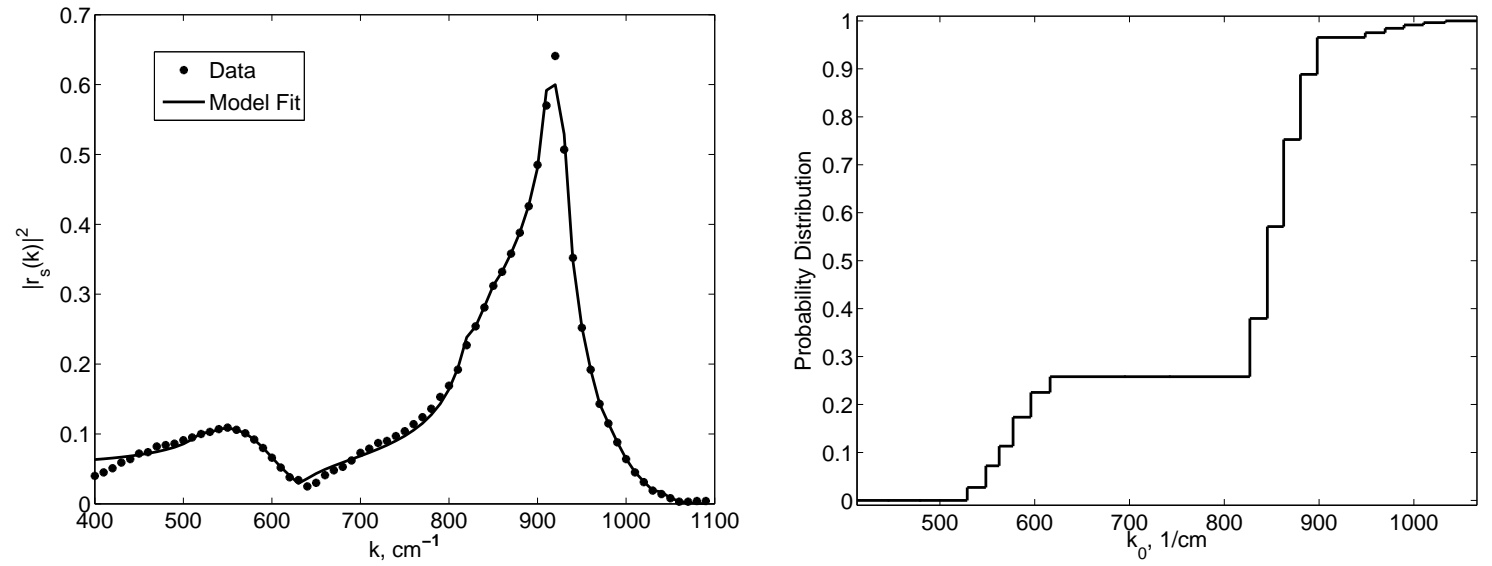

Figure 13: Model fit (left) and the estimated distribution (right) from the full inverse problem (4.3) where $N=20$ for Vitreous Germania.
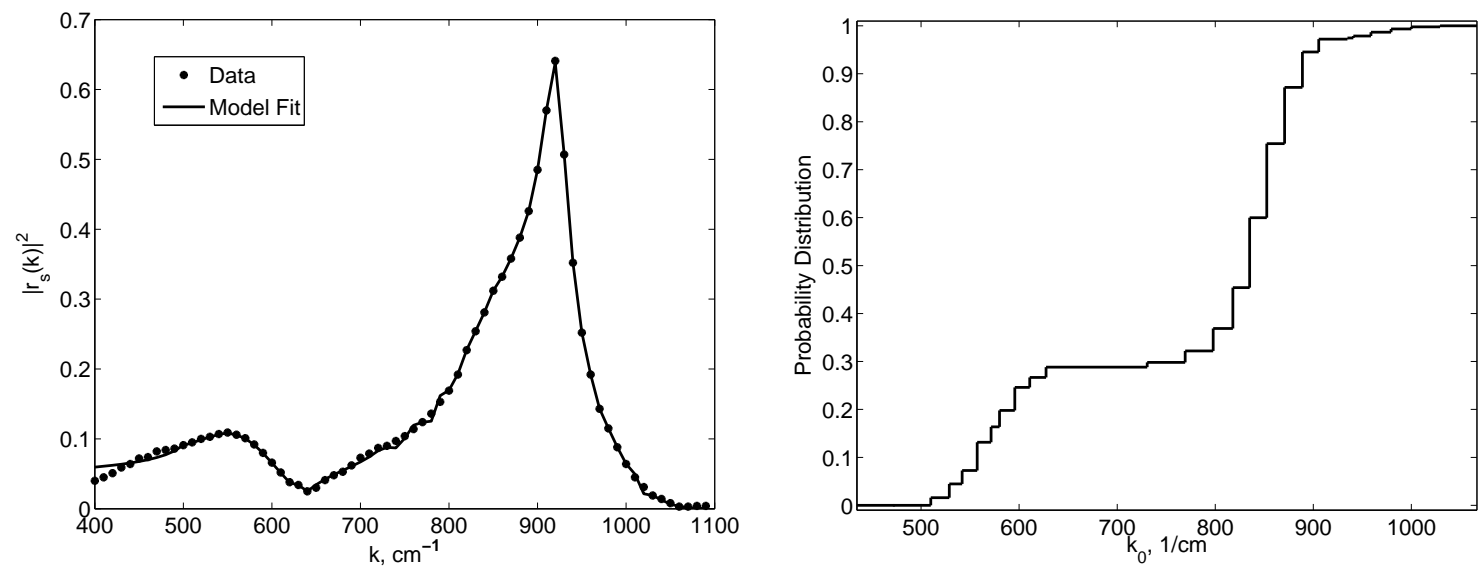

Figure 14: Model fit (left) and the estimated distribution (right) from the full inverse problem (4.3) where $N=25$ for Vitreous Germania. 


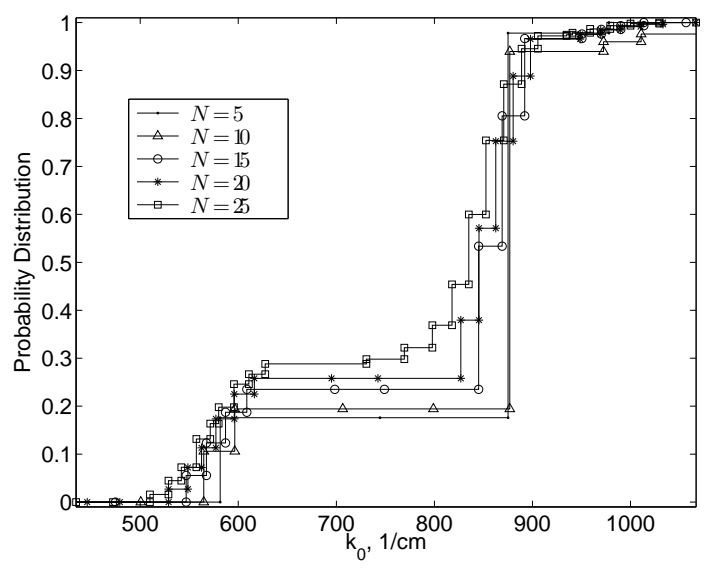

Figure 15: The estimated distributions for all values of $N$ considered from the Vitreous Germania data.

The parameter estimates for $\varepsilon_{s}, \varepsilon_{\infty}$ and $\tau$ are given in Table 2. Since the material was

\begin{tabular}{|c|ccc|}
\hline$N$ & $\varepsilon_{s}$ & $\varepsilon_{\infty}$ & $\tau(\mathrm{cm})$ \\
\hline 5 & 2.7677 & 2.1518 & 0.0275 \\
10 & 2.5768 & 1.9634 & 0.0418 \\
15 & 2.5999 & 1.9904 & 0.0525 \\
20 & 2.4677 & 1.8341 & 0.0578 \\
25 & 2.4361 & 1.7732 & 0.0581 \\
\hline
\end{tabular}

Table 2: Estimations obtained using the reflectance data for Vitreous Germania using various numbers of Dirac measures.

not interrogated at wavenumbers lower than $400 \mathrm{~cm}^{-1}$, we cannot expect to reliably estimate the "static" dielectric constant $\varepsilon_{s}$. With this in mind, we see that the estimated value of $\varepsilon_{\infty}$ generally decreases as $N$ increases. With the values of $N=20$ and 25 , the estimates for $\varepsilon_{\infty}$ are relatively close to each other. Similarly, the estimates for $\tau$ increase as $N$ increases, and above the value of $N=15$ at which the model better fits the data, the values for $\tau$ remain at approximately $0.05+$. This may suggest that once a sufficient number of nodes are used to provide a "good" model fit, the parameter estimates settle around stationary values even as $N$ continues to increase. Again, we emphasize that the ideal number of nodes to use is a topic for further research and the corresponding solution will undoubtedly be dependent on the inherent underlying (and most likely unknown) properties of the material, specifically, the form of the underlying distribution of resonance wavenumbers.

As a second consideration, we solve the optimization problem using reflectance data obtained from Vitreous Silica (available in Table A3 in [23]) over the 200-900 $\mathrm{cm}^{-1}$ range. The resulting model fits and estimated distributions are given in Figures 16-20. Here, we again see that the model fits improve as $N$ increases, and by $N=25$ an excellent fit is obtained. As before, we plot the distributions obtained using different values of $N$ on one graph in Figure 21. From this we see that all of the estimated distributions agree quite well with each other, with a major jump to 0.6 at around $450 \mathrm{~cm}^{-1}$ and a second jump of 0.4 at approximately $1080 \mathrm{~cm}^{-1}$. 

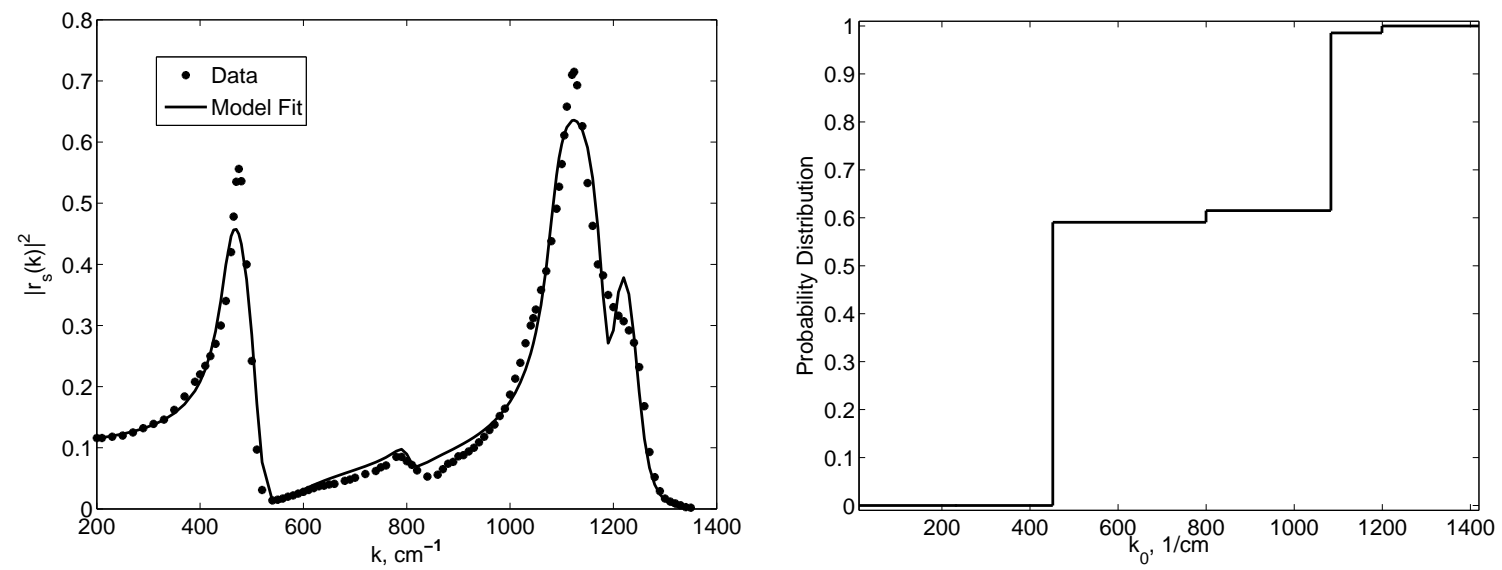

Figure 16: Model fit (left) and the estimated distribution (right) from the full inverse problem (4.3) where $N=5$ for Vitreous Silica.
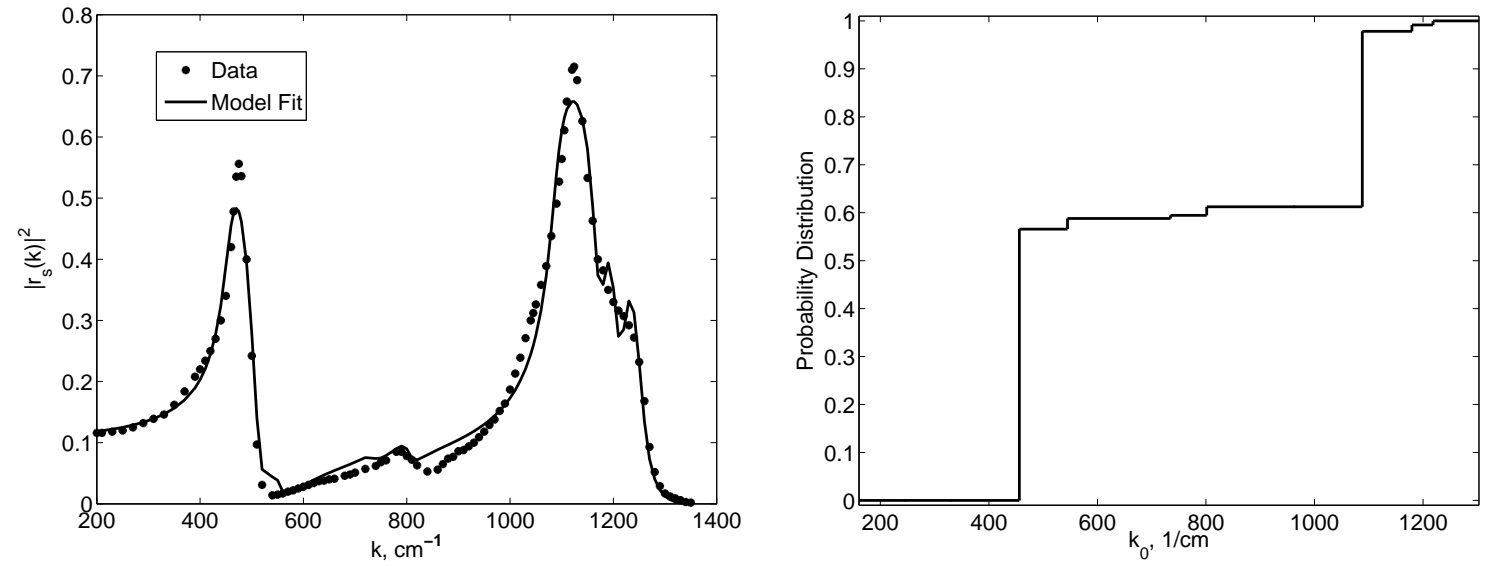

Figure 17: Model fit (left) and the estimated distribution (right) from the full inverse problem (4.3) where $N=10$ for Vitreous Silica.
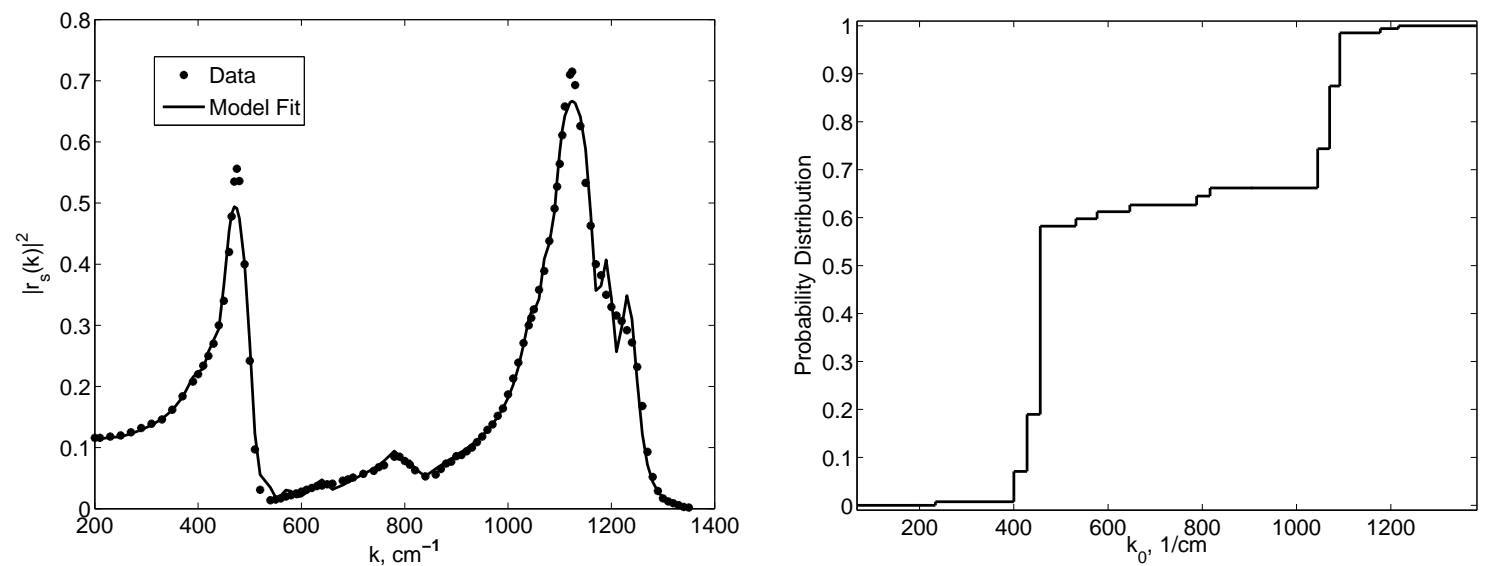

Figure 18: Model fit (left) and the estimated distribution (right) from the full inverse problem (4.3) where $N=15$ for Vitreous Silica. 

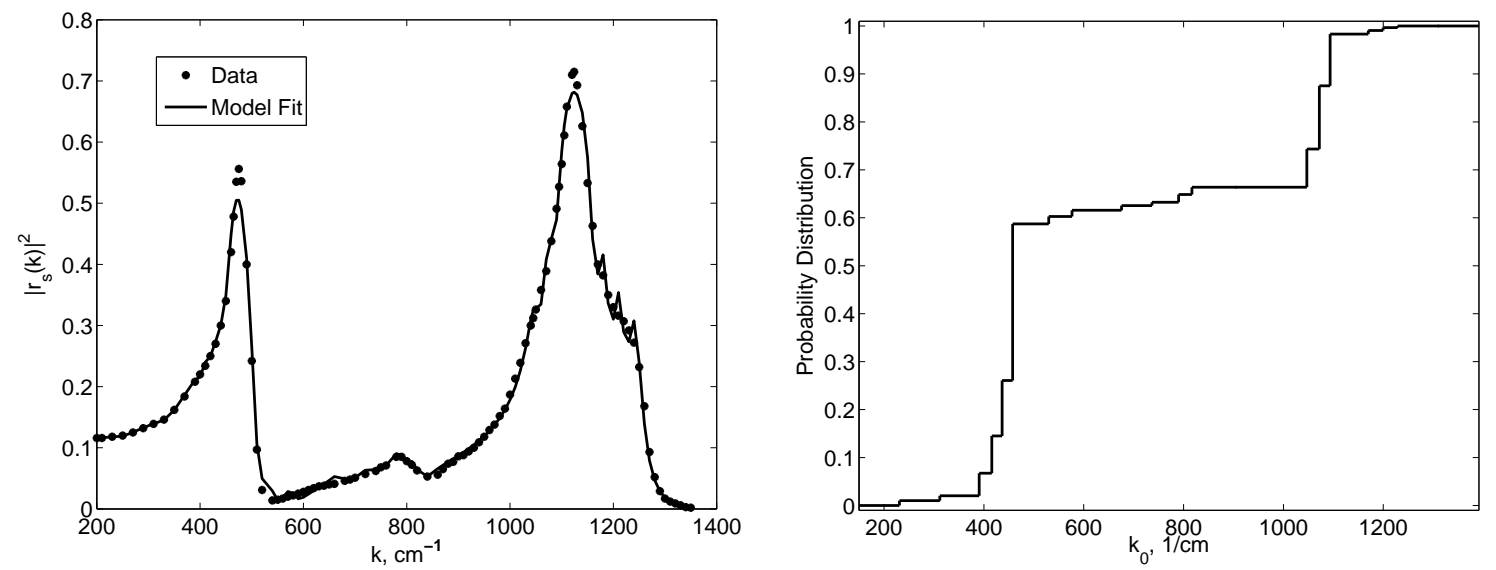

Figure 19: Model fit (left) and the estimated distribution (right) from the full inverse problem (4.3) where $N=20$ for Vitreous Silica.
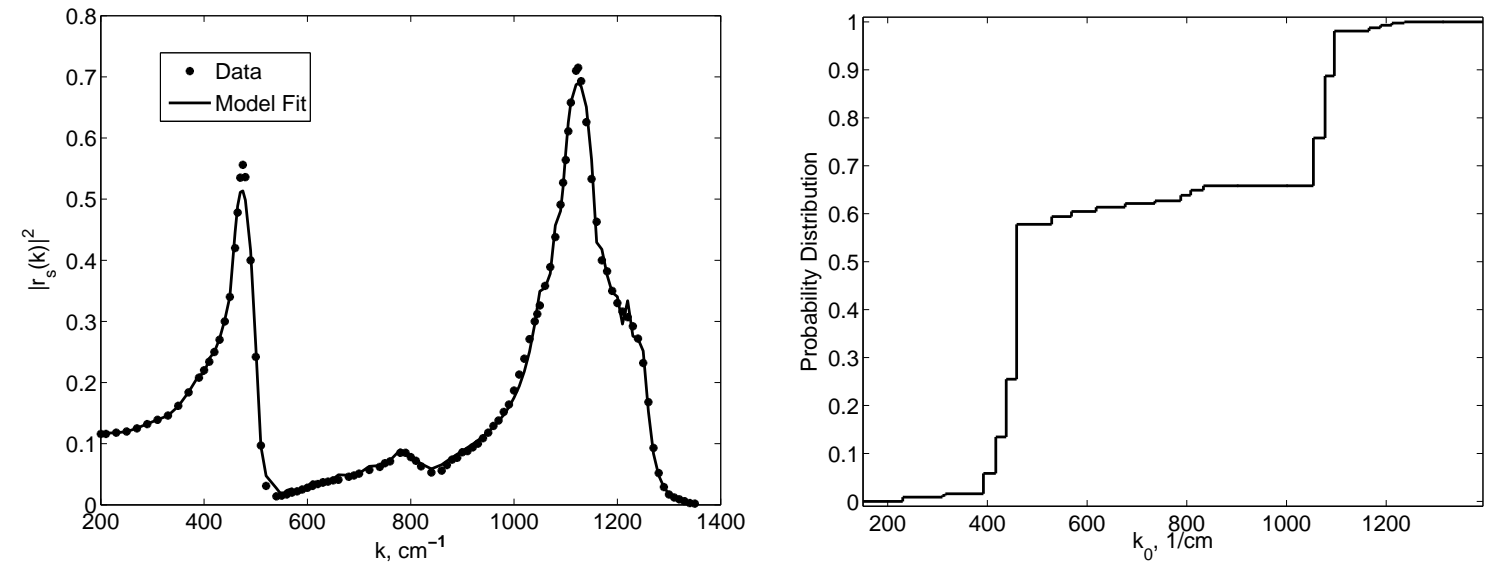

Figure 20: Model fit (left) and the estimated distribution (right) from the full inverse problem (4.3) where $N=25$ for Vitreous Silica.

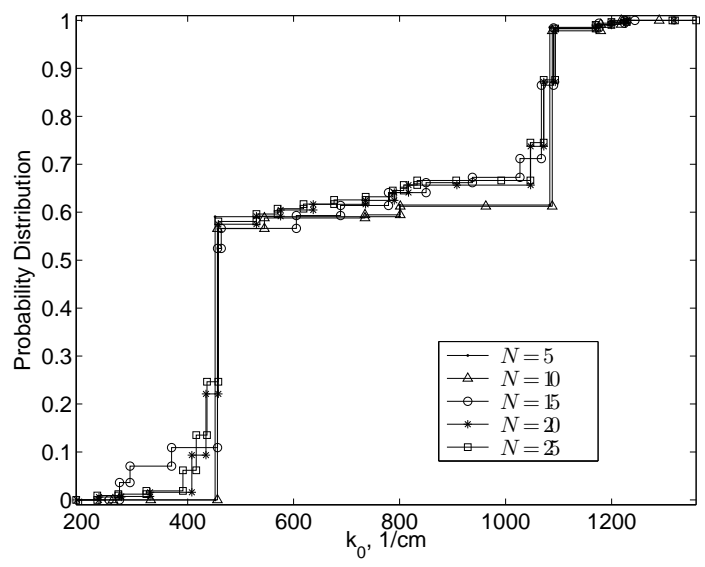

Figure 21: The estimated distributions for all values of $N$ considered from the Vitreous Silica data. 
In Table 3 we present the estimated parameter values obtained. For the glass Vitreous Silica, baseline values for $\varepsilon_{s}$ and $\varepsilon_{\infty}$ which were obtained experimentally are available in [27]. This gives a general benchmark to determine if our estimates for these values are valid. In fact, our estimated values are in very good agreement with the experimental values, particularly for $N=20$, and 25 which, not surprisingly, provide the best model fits to the data.

\begin{tabular}{|c|ccc|}
\hline$N$ & $\varepsilon_{s}$ & $\varepsilon_{\infty}$ & $\tau(\mathrm{cm})$ \\
\hline 5 & 3.8636 & 2.2615 & 0.0277 \\
10 & 3.9546 & 2.3613 & 0.0323 \\
15 & 3.9805 & 2.1704 & 0.0341 \\
20 & 3.7402 & 2.0880 & 0.0397 \\
25 & 3.7818 & 2.0879 & 0.0398 \\
\hline Expt. & 3.8 & 2.1 & \\
\hline
\end{tabular}

Table 3: Parameter estimates obtained using the reflectance data for Vitreous Silica employing various numbers of Dirac measures compared to experimental values (abbreviated as Expt. in the table) taken from [27].

\subsection{Results Obtained Using Simulated Data When Estimating a Probability Measure on the Relaxation Time}

In this section we turn our focus to estimating a distribution of relaxation times rather than resonance wavenumbers. Therefore, in the remainder of this section we take $\theta=\left(\varepsilon_{s}, \varepsilon_{\infty}, k_{0}\right)^{T}$, and denote the nodes of the Dirac masses as $\xi_{m}=\tau_{m}$. The data was generated by evaluating (3.6) with $h$ given by (3.2), where $k_{j}=\omega_{j}$, with $\left\{\omega_{j}\right\}_{j=0}^{100}=\left\{10^{7+0.09 j}\right\}_{j=0}^{100}$, and $\nu_{j}$ was chosen as a realization of a normally distributed random variable with mean 0 and standard deviation $\sigma_{0}=0.001$. The true probability measure $G_{0}$ was chosen as

$$
G_{0}=0.6 \Delta_{\tau_{k}^{1}}+0.4 \Delta_{\tau_{k}^{2}}
$$

where $\tau_{k}^{1}=2 \pi c \cdot 7.14 \times 10^{-16}$ and $\tau_{k}^{2}=2 \pi c \cdot 10^{-14}$, where $c$ is the speed of light in $\mathrm{cm} / \mathrm{s}$. We used the true parameters $\theta_{0}=\left(2.25,1.25, k_{0}\right)^{T}$, where $k_{0}=\omega_{0} /(2 \pi c), \omega_{0}=10^{14}$. We note that these choices for frequency ranges and parameter values are adapted from [17]. To keep the scenario simple, we take $\theta_{0}$ to be known and only estimate the probability measure.

We first consider the case where the nodes are kept fixed and choose $\left\{\tau_{m}\right\}_{m=1}^{N}$ to be $N$ evenly spaced nodes over the interval $2 \pi c \cdot\left[10^{-17}, 2 \times 10^{-14}\right]$. In Figure 22 we give the model fit and the estimated probability measure for $N=15$. Although the model fit is reasonable, the estimated distribution is nowhere near the true distribution. As we did in the case of estimating a distribution of wavenumbers, we will attempt to resolve this issue by optimizing the node locations as well. In Figure 23 we give the results where both the weights and the locations of the Dirac masses have been estimated. Compared to the case of fixed nodes, the model fit and the estimated distribution are both improved. However, the estimated distribution does not recover the single increase in the true distribution at approximately $\tau \approx 2 \mathrm{~cm}$. Rather, the estimated distribution approximates the jump by slowly increasing from 0.6 at $\tau \approx 1.5 \mathrm{~cm}$ to 1 at $\tau \approx 2.25 \mathrm{~cm}$. 

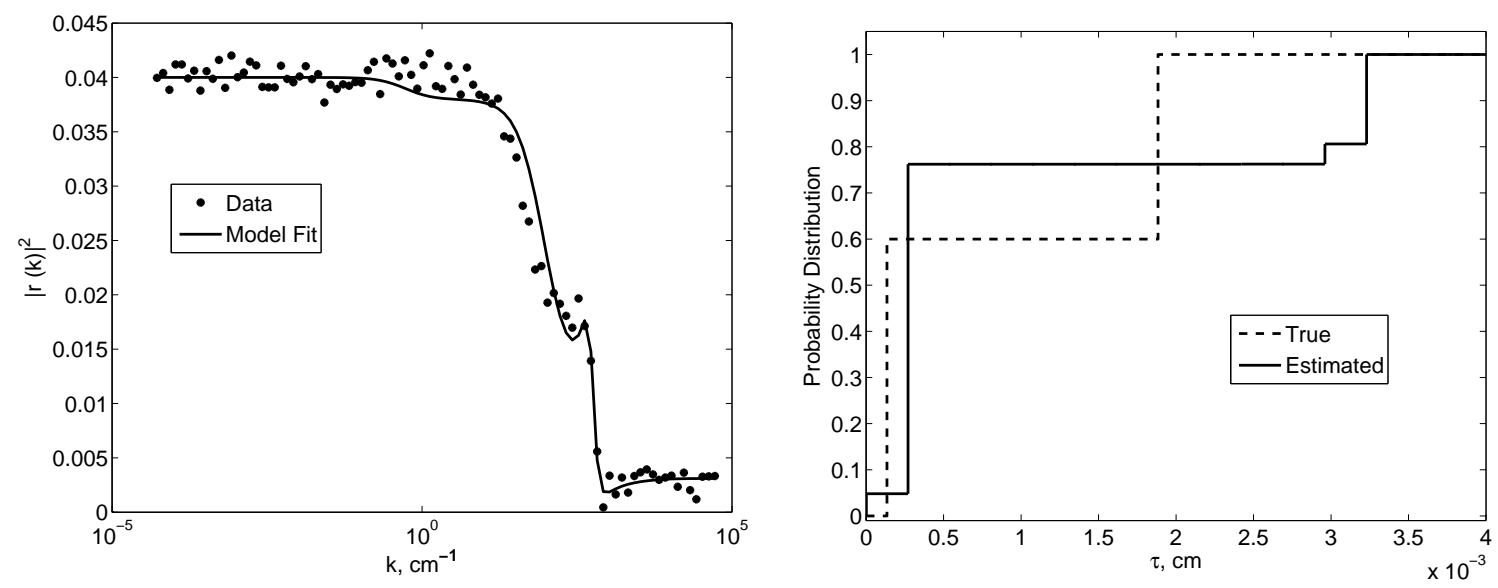

Figure 22: Model fit (left), and the estimated distribution (right) with $N=15$ fixed nodes.
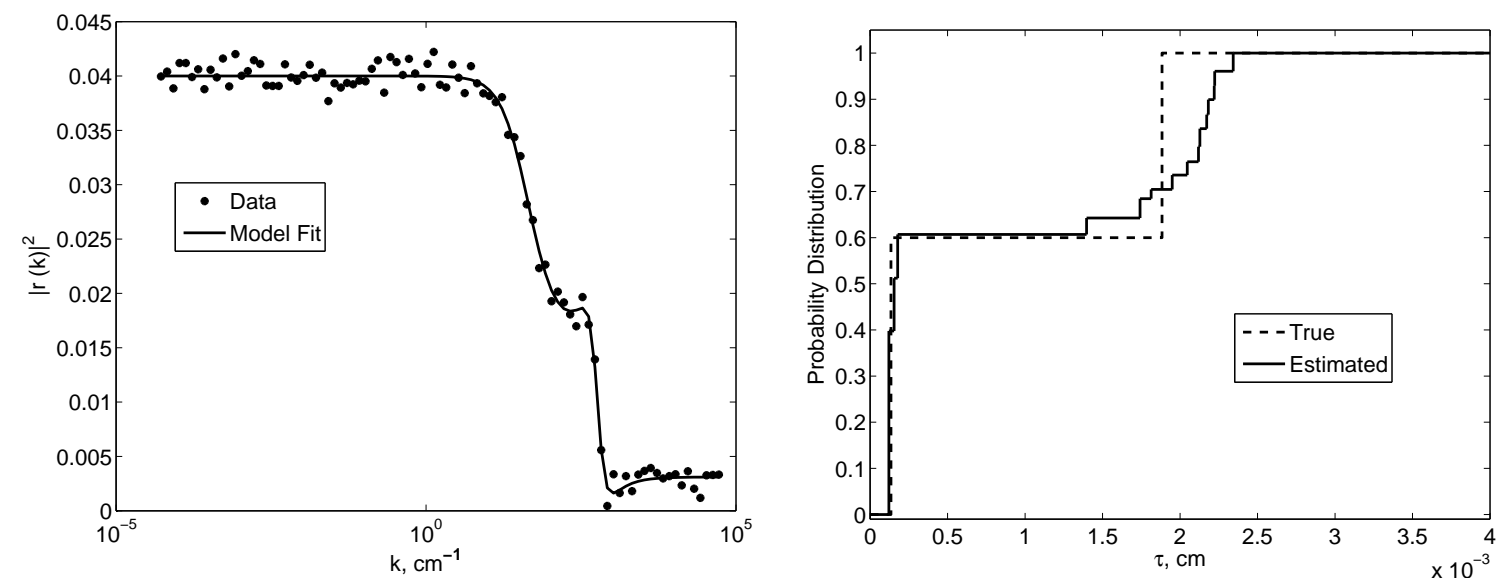

Figure 23: Model fit (left) and the estimated distribution (right) where both $N=15$ node weights and locations were optimized.

Finally, we consider the addition of derivative information into the minimization problem, hence, we take $h$ as in (3.3). As before, both the node weights and the locations are being estimated. In Figure 24 we give the model and derivative fits, as well as the estimated distribution. The model fit is again quite good, and the derivative fit is reasonable. However, in this case, we are able to recover, almost precisely the true distribution. 

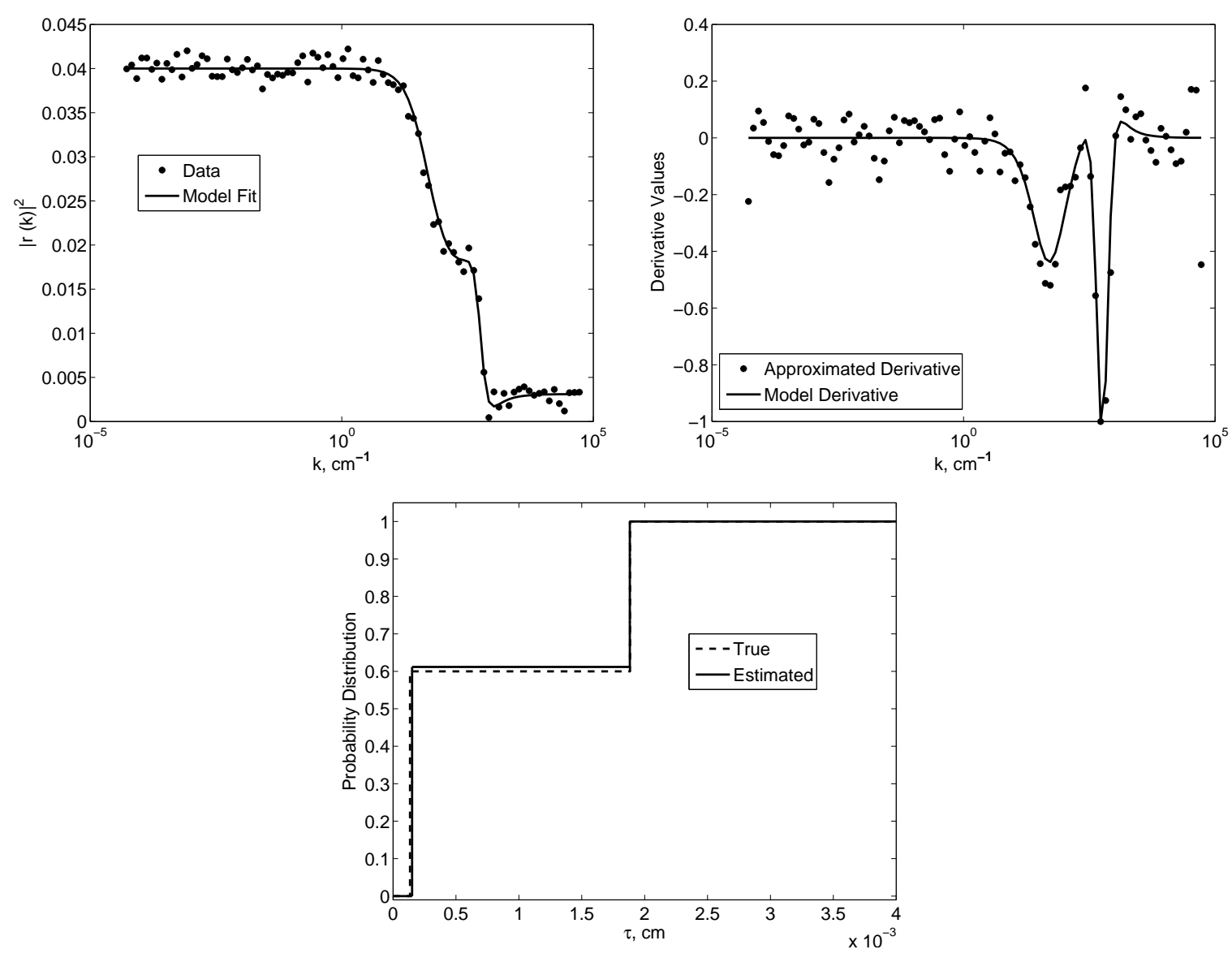

Figure 24: Model fit (left), the derivative of reflectance fit (right), and the estimated distribution (bottom) where both $N=15$ node weights and locations were optimized.

\section{Concluding Remarks and Future Research Efforts}

In this presentation we imposed a probability measure on the resonance wavenumber as well as the relaxation time within the Lorentz polarization model. We then summarized an existing computational framework developed by our group to non-parametrically estimate the unknown probability measure. We carried out existing Dirac measure approximation methods on a set of simulated data to illustrate the basic feasibility and restrictions of these methods for this particular application. We then improved the existing approximation methods by optimizing both weights and nodes of the approximating Dirac measures, and demonstrated the capability of the proposed methods to accurately recover a true distribution for the simulated data. Additionally, we applied our methods of estimating a distribution of resonance wavenumbers to available data sets of reflectance collected from inorganic glass. Using these data sets we were able to obtain very good fits to the data. The fits improved as the number of nodes $N$ increased, and the estimated distributions and parameter values appeared to stabilize as the value of $N$ was increased. When possible, we showed that our parameter estimates agreed well with available experimental values. Finally, using simulated data we showed that when estimating a distribution of relaxation times the use of derivative measurements increases the accuracy of the method.

Future efforts may include using a model selection criteria, such as the Akaike Informa- 
tion Criterion, in order to quantitatively determine an optimal number of Dirac measures. Alternatively, one could devise a pseudo "mesh refinement" in which initially a low number of nodes is chosen, then during the optimization process more nodes are added until a desired accuracy is reached. Moreover, for particular applications, it may be necessary to simultaneously estimate a probability measure on the resonance wavenumbers and the relaxation times. Thus, our methods would require extension to such a case. It may also prove useful to investigate the robustness of the estimation procedure we have outlined on simulated data generated from a continuous, rather than discrete distribution. Moreover, one may wish to extend these results to the case where the unknown probability measure is known to be absolutely continuous so that the corresponding probability density function (PDF) exists and can be approximated by piecewise linear spline functions (see [14] for details). It was demonstrated in [8] that for such a case the spline-based approximation methods converge much faster than do the Dirac measure approximation methods and they also provide convergence for the associated PDFs while the Dirac measure approximation methods do not do this.

Another future effort involves quantifying uncertainties of the probability estimators obtained using this modified Dirac measure approximation method. The pointwise asymptotic normality result for the approximated probability measure estimators was established in our theoretic companion paper [7] for the case where the original Dirac measure approximation method is used for approximation. It was based on the asymptotic normality results for the corresponding weights of the approximated probability measure estimator under the model "misspecification" due to approximation as well as the fact that the approximated probability measure estimator is linearly dependent on these weights. Numerical results demonstrated in [7] show that one has a good match for the confidence bands obtained by the pointwise asymptotic normality result and the Monte Carlo method in the region to which the model output is most sensitive. However, the pointwise asymptotic normality result established in [7] cannot be applied to the case where the modified Dirac measure approximation method is used as the obtained approximated probability measure estimator is not linearly dependent on its corresponding weights and node locations.

\section{Acknowledgements}

This research was supported in part by Grant Number NIAID R01AI071915-10 from the National Institute of Allergy and Infectious Diseases, in part by the Air Force Office of Scientific Research under grant number AFOSR FA9550-12-1-0188, in part by the Army Research Office under contract number W911NF-13-P-0017, in part by the National Science Foundation under Research Training Grant (RTG) DMS-0636590 and in part by the US Department of Education Graduate Assistance in Areas of National Need (GAANN) under grant number P200A120047. The authors are grateful to Bill Browning, Amanda Criner and Katie Leonard for helpful discussions during the course of parts of the research reported here. 


\section{References}

[1] P. Baldus, M. Jansen and D. Sporn, Ceramic Fibers for Matrix Composites in HighTemperature Engine Applications, Science, 285 (1999), 699-703.

[2] H.T. Banks, A Functional Analysis Framework for Modeling, Estimation and Control in Science and Engineering, Chapman and Hall/CRC Press, Boca Raton, FL, 2012.

[3] H.T. Banks and K.L. Bihari, Modeling and estimating uncertainty in parameter estimation, Inverse Problems, 17 (2001), 95-111.

[4] H.T. Banks, V.A. Bokil and N.L. Gibson, Parameter estimation versus homogenization techniques in time-domain characterization of composite dielectrics, J. Inv. Ill-Posed Probs., 15 (2007), 19-37.

[5] H.T. Banks, V.A. Bokil and N.L. Gibson, Analysis of Stability and Dispersion in a Finite Element Method for Debye and Lorentz Dispersive Media, CRSC Technical Report CRSC-TR06-21, North Carolina State University, 2006; Numer. Methods for Partial Diff. Eqs., 25.4 (July 2009) 885-917.

[6] H.T. Banks, M.W. Buksas and T. Lin, Electromagnetic Material Interrogation Using Conductive Interfaces and Acoustic Wavefronts, Frontiers in Applied Mathematics, Vol. FR21, SIAM, Philadelphia, PA, 2000.

[7] H. T. Banks, J. Catenacci, and S. Hu, Asymptotic properties of probability measure estimators in a nonparametric model, CRSC-TR14-05, N. C. State University, Raleigh, NC, May, 2014; SIAM/ASA Journal on Uncertainty Quantification, submitted. .

[8] H.T. Banks and J.L. Davis, A comparison of approximation methods for the estimation of probability distributions on parameters, Applied Numerical Mathematics, 57 (2007), $753-777$.

[9] H.T. Banks and B.G. Fitzpatrick, Estimation of growth rate distributions in sizestructured population models, Quarterly of Applied Mathematics, 49 (1998), 215-235.

[10] H.T. Banks, B.G. Fitzpatrick, L.K. Potter and Y. Zhang, Estimation of probability distributions for individual parameters using aggregate population data, CRSC-TR98-6, January, 1998; In Stochastic Analysis, Control, Optimization and Applications, (Edited by W. McEneaney, G. Yin and Q. Zhang), Birkhäuser Verlag, Basel, 1998, 353-371.

[11] H.T. Banks and N.L. Gibson, Electromagnetic inverse problems involving distributions of dielectric mechanisms and parameters, Quarterly of Applied Mathematics, 64 (2006), 749-795.

[12] H.T. Banks, S. Hu and W.C. Thompson, Modeling and Inverse Problems in the Presence of Uncertainty, Taylor/Francis-Chapman/Hall-CRC Press, Boca Raton, FL, 2014.

[13] H.T. Banks, Z.R. Kenz, and W.C. Thompson, A review of selected techniques in inverse problem nonparametric probability distribution estimation, J. Inverse and Ill-Posed Problems, 20 (2012), 429-460. 
[14] H.T. Banks and G.A. Pinter, A probabilistic multiscale approach to hysteresis in shear wave propagation in biotissue, SIAM J. Multiscale Modeling and Simulation, 3 (2005), 395412.

[15] H.T. Banks and H.T. Tran, Mathematical and Experimental Modeling of Physical and Biological Processes, CRC Press, Boca Raton, FL, 2009.

[16] P. Billingsley, Convergence of Probability Measures, Wiley \& Sons, New York, 1968.

[17] J.G. Blaschak and J. Fanzen, Precursor propagation in dispersive media from shortrise-time pulses at oblique incidence, Journal of the Optical Society of America A, 12 (1995), 1501-1512.

[18] Bruker OPUS spectroscopic software reference manual, version 4.2, 2003.

[19] K.P. Burnham and D.R. Anderson, Model Selection and Inference: A Practical Information-Theoretical Approach, 2nd edition, Springer-Verlag, New York, 2002.

[20] A.T. Cooney and R.Y. Flattum-Riemers, Non-destructive evaluation of degradation in EB-PVD barrier coatings by infrared reflectance spectroscopy, AIP Conference Proceedings, Denver, Colorado, July 15-20, 2012.

[21] A.T. Cooney, R.Y. Flattum-Riemers, and B.J. Scott, Characterization of material degradation in ceramic matrix composites using infrared reflectance spectroscopy, AIP Conference Proceedings, San Diego, California, July 18-23, 2010.

[22] R.M. Dudley, Real Analysis and Probability, Cambridge University Press, Cambridge, UK, 2002.

[23] A.M. Efimov, Optical Contants of Inorganic Glasses, CRC press, Boca Raton, Florida, 1995.

[24] A.M. Efimov, Quantitative IR spectroscopy: Applications to studying glass structure and properties, Journal of Non-Crystalline Solids, 203 (1996), 1-11.

[25] A.M. Efimov, Vibrational spectra, related properties, and structure of inorganic glasses, Journal of Non-Crystalline Solids, 253 (1999), 95-118.

[26] J.I. Eldridge, C.M. Spuckler and R.E. Martin, Monitoring delamination progression in thermal barrier coatings by mid-infrared reflectance imaging, International Journal of Applied Ceramic Technology, 3 (2006), 94-104.

[27] L. Giacomazzi and A. Pasquarello, Vibrational spectra of vitreous $\mathrm{SiO}_{2}$ and vitreous $\mathrm{GeO}_{2}$ from first principles, Journal of Physics: Condensed Matter, 19 (2007), 112-121.

[28] N.L. Gibson, Terahertz-Based Electromagnetic Interrogation Techniques for Damage Detection, PhD Thesis, NCSU, Raleigh, 2004.

[29] D.J. Griffiths, Introduction to Electrodynamics, 3rd edition, Prentice Hall, Upper Saddle River, New Jersey, 1999. 
[30] D. Lesnic, G. Wakefield, B.D. Sleeman, and J.R. Ockendon, Determination of the index of refraction of anti-reflection coatings, MICS Journal, 2 (2010), 155-173.

[31] S.R. Pina, L.C. Pardini, I.V. Yoshida, Carbon fiber/ceramic matrix composites: processing, oxidation and mechanical properties, Journal of Material Science, 42 (2007) $4245-4253$.

[32] Yu.V. Prohorov, Convergence of random processes and limit theorems in probability theory, Theor. Prob. Appl., 1 (1956), 157-214.

[33] G. Qi, C. Zhang, H. Hu, F. Cao, S. Wang, Y. Jiang, B. Li, Crystallization behavior of three-dimensional silica fiber reinforced silicon nitride composite, Journal of Crystal Growth, 284 (2005), 293-296.

[34] H. Ohnabe, S. Masaki, M. Onozuka, K. Miyahara, T. Sasa, Potential application of ceramic matrix composites to aero-engine components, Composites Part A: Applied Sciences and Manufacturing, 30 (1999), 489-496.

[35] V. Raman, G. Bhatia, P.R. Sengupta, A.K. Srivastava, K.N. Sood, Synthesis of silicon carbide nanorods from mixture of polymer and sol-gel silica, Journal of Material Science, 42 (2007), 5891-5895.

[36] E.J. Rothwell and M.J. Cloud, Electromagnetics, CRC Press, LLC, 2001. 